\title{
$\tau$-REGULAR FACTORIZATION IN COMMUTATIVE RINGS WITH ZERO-DIVISORS
}

\author{
CHRISTOPHER PARK MOONEY
}

\begin{abstract}
Recently there has been a flurry of research on generalized factorization techniques in both integral domains and rings with zero-divisors, namely, $\tau$-factorization. There are several ways that authors have studied factorization in rings with zero-divisors. This paper focuses on the method of regular factorizations introduced by Anderson and Valdes-Leon. We investigate how one can extend the notion of $\tau$-factorization to commutative rings with zero-divisors by using the regular factorization approach. The study of regular factorization is particularly effective because the distinct notions of associate and irreducible coincide for regular elements. We also note that the popular U-factorization developed by Fletcher also coincides since every regular divisor is essential. This will greatly simplify many of the cumbersome finite factorization definitions that exist in the literature when studying factorization in rings with zero-divisors.
\end{abstract}

1. Introduction. There has been a considerable amount of research done on the factorization properties of commutative rings, especially domains. For various aspects of the theory see $[\mathbf{3}, \mathbf{1 3}, \mathbf{2 2}, \mathbf{2 7}, \mathbf{2 8}, \mathbf{3 2}]$. Unique factorization domains (UFDs) are well understood and have been studied extensively over the years. More recently, many authors have studied rings which satisfy various weakenings of the UFD conditions. These factorization properties of domains have been extended, in several distinct ways to rings with zero-divisors. Traditionally, in the domain case, authors have studied prime or irreducible factorizations. More recently, research has been done on generalizing the types of factorizations that have been studied to include things like co-maximal factorizations or using $\star$-operations to generalize factorization.

2010 AMS Mathematics subject classification. Primary 13A05, 13E99, 13 F15.

Keywords and phrases. Factorization, zero-divisors, commutative rings, regular and U-factorization.

Received by the editors on December 16, 2013, and in revised form on August 13, 2014. 
Of particular interest to the current article is the work of Anderson and Frazier. This is a survey article, [7], on the study of factorization in domains in which the authors introduce $\tau$-factorization. The use of $\tau$-factorization yields a beautiful synthesis of many of these generalizations of factorizations studied in the integral domain case. In many ways, this article was able to consolidate all of the factorization research in integral domains into a single method of studying factorization. Recently, the author has begun to study methods of extending this powerful approach of $\tau$-factorization to the case of a commutative ring with zero-divisors. Because of the numerous approaches that have been taken to study factorization in rings with zero-divisors, this has led to many approaches to extending $\tau$-factorization.

The theory of factorization in commutative rings with zero-divisors was initiated by Anderson, et al., [1, 6, 8, 9, 10] and has been continued by many authors. Recently, there has been an explosion of different approaches, by many different authors, of studying the arithmetic of rings with zero-divisors. For instance, see the work of Chang, Frei, Frisch, Geroldinger, Smertnig, et al., in [21, 26, 29].

In [42], the author used the methods established by Anderson and Valdes-Leon in [9] to extend many of the $\tau$-factorization definitions to also work in rings with zero-divisors. In [43], the author investigated extending $\tau$-factorization using the notion of U-factorizations first developed by Fletcher in $[\mathbf{2 4}, \mathbf{2 5}$ ] and then studied extensively by Axtell, Forman, Roersma and Stickles in [11, 12]. In [44], the author studied yet another approach to extending $\tau$-factorization, by using complete factorizations which was touched on in [7] in the case of integral domains.

In the present article, we concentrate on the approach studied in [10, Section 5] in which Anderson and Valdes-Leon study what was called regular factorization. This approach takes advantage of the fact that, for regular elements, all of the traditionally distinct associate relations and irreducible elements behave as they do in integral domains, where they are all equivalent once again. We see that this approach will greatly simplify matters and in fact unifies many of the previous methods in $[42,43]$.

In Section 2, we provide some necessary background definitions and theorems. In Section 3, we develop many of the definitions of $\tau$-regular- 
factorization, $\tau$-regular irreducible elements as well as $\tau$-regular finite factorization properties that rings may have. This is done by using the approach of Anderson and Valdez-Leon in [10, Section 5], where they restrict their study of $\tau$-factorization to the regular elements of a commutative ring with 1 . In subsection 3.2 , we prove several theorems which describe the relationships between the various $\tau$-regular finite factorization properties that rings may possess. In Section 4, we compare this new method of extending $\tau$-factorization with the previous work in [42] and the relation $\tau_{r}:=\tau \cap \operatorname{Reg}(R) \times \operatorname{Reg}(R)$. In Section 5 , we demonstrate how these $\tau$-regular finite factorization properties are related to other finite factorization properties defined in other works, especially $[42,43]$. Finally, in Section 6 , we study the $\tau$-regular factorization properties of two particular rings that have been of much interest recently. These rings are the self-idealization of a PID, $R(D)$ in [21] and $\mathbb{Z}_{p^{n}}[X]$ studied in [26]. We see which of the $\tau$ factorization, $\tau$-U-factorization and $\tau$-regular factorization properties studied in $[42,43]$ and the present paper are satisfied by these rings. We hope this motivates some future questions to be answered about an even larger class of rings, such as Mori, v-Marot, Krull and C-rings, that have been of interest in the literature on commutative rings with zero-divisors.

2. Preliminary definitions and results. We will assume $R$ is a commutative ring with $1 \neq 0$. Let $R^{*}=R-\{0\}$, let $U(R)$ be the set of units of $R$ and let $R^{\#}=R^{*}-U(R)$ be the non-zero, non-units of $R$. As in [9], we let $a \sim b$ if $(a)=(b), a \approx b$ if there exists $\lambda \in U(R)$ such that $a=\lambda b$, and $a \cong b$ if

(1) $a \sim b$

and

(2) $a=b=0$ or, if $a=r b$ for some $r \in R$, then $r \in U(R)$.

We say $a$ and $b$ are associates (respectively strong associates, very strong associates) if $a \sim b$ (respectively $a \approx b, a \cong b$ ). As in [5], a ring $R$ is said to be strongly associate (respectively very strongly associate) ring if for any $a, b \in R, a \sim b$ implies $a \approx b$ (respectively $a \cong b$ ).

2.1. $\tau$-factorization in rings with zero-divisors. Let $\tau$ be a relation on $R^{\#}$, that is, $\tau \subseteq R^{\#} \times R^{\#}$. We will always assume further that 
$\tau$ is symmetric. For non-units $a, a_{i} \in R$, and $\lambda \in U(R), a=\lambda a_{1} \cdots a_{n}$ is said to be a $\tau$-factorization if $a_{i} \tau a_{j}$ for all $i \neq j$. If $n=1$, then this is said to be a trivial $\tau$-factorization. Given the above $\tau$-factorization, we would say that $a_{i}$ is a $\tau$-factor of $a$ or write $\left.a_{i}\right|_{\tau} a$.

As in [42], we say $\tau$ is multiplicative (respectively divisive) if for $a, b, c \in R^{\#}$ (respectively $a, b, b^{\prime} \in R^{\#}$ ), $a \tau b$ and $a \tau c$ imply $a \tau b c$ (respectively $a \tau b$ and $b^{\prime} \mid b$ imply $a \tau b^{\prime}$ ). We say $\tau$ is associate (respectively strongly associate, very strongly associate) preserving if, for $a, b, b^{\prime} \in R^{\#}$ with $b \sim b^{\prime}$ (respectively $b \approx b^{\prime}, b \cong b^{\prime}$ ) $a \tau b$ implies $a \tau b^{\prime}$. A $\tau$-refinement of a $\tau$-factorization $\lambda a_{1} \cdots a_{n}$ is a factorization of the form,

$$
\left(\lambda \lambda_{1} \cdots \lambda_{n}\right) b_{11} \cdots b_{1 m_{1}} \cdot b_{21} \cdots b_{2 m_{2}} \cdots b_{n 1} \cdots b_{n m_{n}},
$$

where $a_{i}=\lambda_{i} b_{i_{1}} \cdots b_{i_{m_{i}}}$ is a $\tau$-factorization for each $i$. We say that $\tau$ is refinable if every $\tau$-refinement of a $\tau$-factorization is a $\tau$-factorization. We say $\tau$ is combinable if whenever $\lambda a_{1} \cdots a_{n}$ is a $\tau$-factorization, then so is each $\lambda a_{1} \cdots a_{i-1}\left(a_{i} a_{i+1}\right) a_{i+2} \cdots a_{n}$.

We now pause to supply the reader with a few examples of particularly useful or interesting $\tau$-relations to give an idea of the power of $\tau$-factorization.

Example 2.1. Let $R$ be a commutative ring with 1 .

(i) $\tau=R^{\#} \times R^{\#}$. This yields the usual factorizations in $R$ and $\left.\right|_{\tau}$ is the same as the usual divides. $\tau$ is multiplicative and divisive (hence associate preserving as we shall soon see).

(ii) $\tau=\emptyset$. For every $a \in R^{\#}$, there is only the trivial factorization and $a \mid{ }_{\tau} b \Leftrightarrow a=\lambda b$ for $\lambda \in U(R) \Leftrightarrow a \approx b$. Again, $\tau$ is both multiplicative and divisive (vacuously).

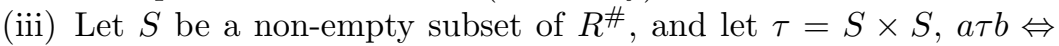
$a, b \in S$. So $\tau$ is multiplicative (respectively divisive) if and only if $S$ is multiplicatively closed (respectively closed under nonunit factors). A non-trivial $\tau$-factorization is up to unit factors a factorization into elements from $S$.

(iv) Let $\star$ be a star-operation on $R$ and define $a \tau b \Leftrightarrow(a, b)^{\star}=R$, that is $a$ and $b$ are $\star$-coprime or $\star$-comaximal. This particular operation has been studied more in depth by Juett [33]. When $\star=d$, the identity star operation, we get the co-maximal factorizations of McAdam and Swan [41]. 
(v) Let $a \tau_{z} b \Leftrightarrow a b=0$. Then every $a \in R^{\#}$ is a $\tau$-atom. The only nontrivial $\tau$-factorizations are $0=\lambda a_{1} \cdot \ldots \cdot a_{n}$ where $a_{i} \cdot a_{j}=0$ for all $i \neq j$. This example was studied extensively in [42] and has a close relationship with zero-divisor graphs.

(vi) Let $a \tau b \Leftrightarrow a, b \in \operatorname{Reg}(R)$. Then this gives us the regular factorization studied in [1]. This is the inspiration for Section 3.

(vii) Letting $\tau \subseteq R^{\#} \times R^{\#}$, then we define $\tau_{\text {reg }}:=\tau \cap(\operatorname{Reg}(R) \times$ $\operatorname{Reg}(R))$. Because the collection of regular elements is a saturated, multiplicatively closed set, this has the effect of only allowing trivial factorizations of the zero-divisors. This is the type of $\tau$-factorization we would like to use to compare with the notion of $\tau$-factorizations by way of the regular factorizations studied in [10]. This will be studied more in depth in Section 4.

We now summarize several of the definitions given in $[\mathbf{4 2}, 44]$. Let $a \in R$ be a non-unit. Then $a$ is said to be $\tau$-irreducible or $\tau$-atomic if for any $\tau$-factorization $a=\lambda a_{1} \cdots a_{n}$, we have $a \sim a_{i}$ for some $i$. We will say $a$ is $\tau$-strongly irreducible or $\tau$-strongly atomic if for any $\tau$-factorization $a=\lambda a_{1} \cdots a_{n}$, we have $a \approx a_{i}$ for some $a_{i}$. We will say that $a$ is $\tau$-m-irreducible or $\tau$-m-atomic if for any $\tau$-factorization $a=\lambda a_{1} \cdots a_{n}$, we have $a \sim a_{i}$ for all $i$. Note that the $m$ is for "maximal" since such an $a$ is maximal among principal ideals generated by elements which occur as $\tau$-factors of $a$. As in [44], $a \in R$ is said to be a $\tau$-unrefinable atom if $a$ admits only trivial $\tau$-factorizations. We will say that $a$ is $\tau$-very strongly irreducible or $\tau$-very strongly atomic if $a \cong a$ and $a$ has no non-trivial $\tau$-factorizations. See $[42,44]$ for more equivalent definitions of these various forms of $\tau$-irreducibility.

We have the following relationship between the various types of $\tau$ irreducibles which is proved in [42, Theorem 3.9] as well as [44].

Theorem 2.2. Let $R$ be a commutative ring with 1 , and let $\tau$ be a symmetric relation on $R^{\#}$. Let $a \in R$ be a non-unit. The following diagram illustrates the relationship between the various types of $\tau$ irreducibles and might satisfy where $\approx$ represents $R$ being a strongly associate ring. 


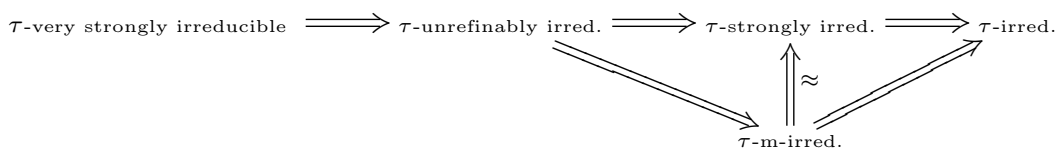

Following Bouvier, a ring $R$ is said to be présimplifiable if $x=x y$ implies $x=0$ or $y \in U(R)$ as in $[14, \mathbf{1 5}, \mathbf{1 6}, \mathbf{1 7}]$. When $R$ is présimplifiable, the various associate relations coincide. As seen in [42], for non-zero elements, if $R$ is présimplifiable, then $\tau$-irreducible will imply $\tau$-very strongly irreducible and the various types of irreducible elements will also coincide. Any integral domain or quasi-local ring is présimplifiable. Examples are given in [9] and abound in the literature which show that in a general commutative ring setting, each of these types of irreducible elements are distinct. For further discussion of the different $\tau$-irreducible elements, the reader is directed to [42].

This leads to the following $\tau$-finite factorization properties that a commutative ring may possess given a particular choice for $\tau$, defined in $[42,44]$. Let $\alpha \in$ atomic, strongly atomic, m-atomic, unrefinably atomic, very strongly atomic $\}, \beta \in\{$ associate, strongly associate, very strongly associate $\}$ and $\tau$ a symmetric relation on $R^{\#}$. Then $R$ is said to be $\tau$ - $\alpha$ if every non-unit $a \in R$ has a $\tau$-factorization $a=\lambda a_{1} \cdots a_{n}$ with $a_{i}$ being $\tau$ - $\alpha$ for all $1 \leq i \leq n$. We will call such a factorization a $\tau$ - $\alpha$-factorization. We say $R$ satisfies the $\tau$ ascending chain condition on principal ideals (ACCP) if for every chain $\left(a_{0}\right) \subseteq\left(a_{1}\right) \subseteq \cdots \subseteq\left(a_{i}\right) \subseteq \cdots$ with $\left.a_{i+1}\right|_{\tau} a_{i}$, there exists an $N \in \mathbb{N}$ such that $\left(a_{i}\right)=\left(a_{N}\right)$ for all $i>N$.

A ring $R$ is said to be a $\tau$ - $\alpha$ - $\beta$-unique factorization ring (UFR) if

(1) $R$ is $\tau$ - $\alpha$ and

(2) for every non-unit $a \in R$ any two $\tau$ - $\alpha$ factorizations $a=\lambda_{1} a_{1} \cdots a_{n}=$ $\lambda_{2} b_{1} \cdots b_{m}$ have $m=n$ and there is a rearrangement so that $a_{i}$ and $b_{i}$ are $\beta$.

A ring $R$ is said to be a $\tau$ - $\alpha$-half factorization ring or half factorial ring (HFR) if

(1) $R$ is $\tau$ - $\alpha$ and 
(2) for every non-unit $a \in R$ any two $\tau$ - $\alpha$-factorizations have the same length.

A ring $R$ is said to be a $\tau$-bounded factorization ring (BFR) if for every non-unit $a \in R$, there exists a natural number $N(a)$ such that for any $\tau$-factorization $a=\lambda a_{1} \cdots a_{n}, n \leq N(a)$. A ring $R$ is said to be a $\tau-\beta$ finite factorization ring (FFR) if for every non-unit $a \in R$ there are only a finite number of non-trivial $\tau$-factorizations up to rearrangement and $\beta$. A ring $R$ is said to be a $\tau-\beta$-weak finite factorization ring (WFFR) if for every non-unit $a \in R$, there are only finitely many $b \in R$ such that $b$ is a non-trivial $\tau$-divisor of $a$ up to $\beta$. A ring $R$ is said to be a $\tau-\alpha$ - $\beta$-divisor finite ring (df ring) if for every non-unit $a \in R$, there are only finitely many $\tau$ - $\alpha \tau$-divisors of $a$ up to $\beta$.

These result in the following diagram accompanying [42, Theorem 4.1] illustrating the relationship between the various $\tau$-finite factorization properties in rings with zero-divisors, where $\nabla$ represents $\tau$ refinable.

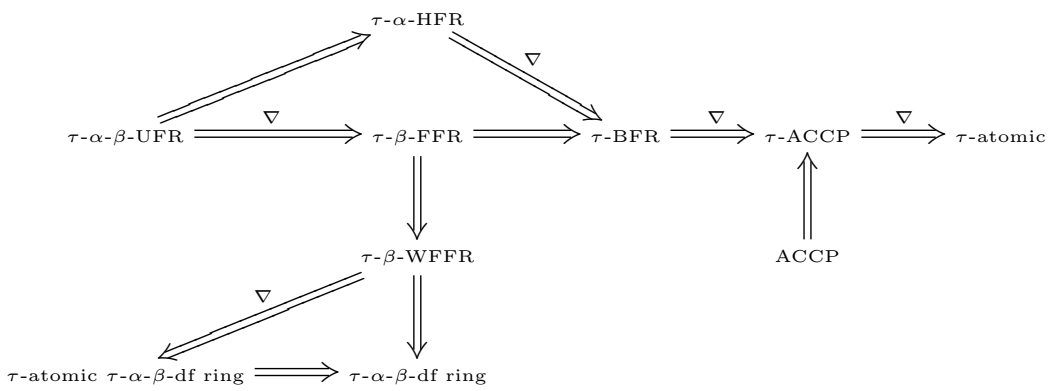

2.2. $\tau$-U-factorization definitions. In this section we briefly present the requisite $\tau$-U-factorization definitions and results from [43]. As in [12], we define U-factorization as follows. Let $a \in R$ be a non-unit. If $a=\lambda a_{1} \cdots a_{n} b_{1} \cdots b_{m}$ is a factorization with $\lambda \in U(R), a_{i}, b_{i} \in R^{\#}$, then we will call

$$
a=\lambda a_{1} a_{2} \cdots a_{n}\left\lceil b_{1} b_{2} \cdots b_{m}\right\rceil
$$

a U-factorization of $a$ if

(1) $a_{i}\left(b_{1} \cdots b_{m}\right)=\left(b_{1} \cdots b_{m}\right)$ for all $1 \leq i \leq n$ and

(2) $b_{j}\left(b_{1} \cdots \widehat{b_{j}} \cdots b_{m}\right) \neq\left(b_{1} \cdots \widehat{b_{j}} \cdots b_{m}\right)$ for $1 \leq j \leq m$ where $\widehat{b_{j}}$ means $b_{j}$ is omitted from the product. 
Here $\left(b_{1} \cdots b_{m}\right)$ is the principal ideal generated by $b_{1} \cdots b_{m}$. The $b_{i}$ 's in this particular U-factorization above will be referred to as essential divisors. The $a_{i}$ 's in this particular U-factorization above will be referred to as inessential divisors. A U-factorization is said to be trivial if there is only one essential divisor.

A $\tau$-U-factorization of a non-unit $a \in R$ is a $\mathrm{U}$-factorization

$$
a=\lambda a_{1} a_{2} \cdots a_{n}\left\lceil b_{1} b_{2} \cdots b_{m}\right\rceil
$$

for which $\lambda a_{1} \cdots a_{n} b_{1} \cdots b_{m}$ is also a $\tau$-factorization.

Given a symmetric relation $\tau$ on $R^{\#}$, we say $R$ is $\tau$-U-refinable if for every $\tau$-U-factorization of any non-unit $a \in U(R)$,

$$
a=\lambda a_{1} \cdots a_{n}\left\lceil b_{1} \cdots b_{m}\right\rceil,
$$

any $\tau$-U-factorization of an essential divisors,

$$
b_{i}=\lambda^{\prime} c_{1} \cdots c_{n^{\prime}}\left\lceil d_{1} \cdots d_{m^{\prime}}\right\rceil
$$

satisfies

$$
a=\lambda \lambda^{\prime} a_{1} \cdots a_{n} c_{1} \cdots c_{n^{\prime}}\left\lceil b_{1} \cdots b_{i-1} d_{1} \cdots d_{m^{\prime}} b_{i+1} \cdots b_{m}\right\rceil
$$

is a $\tau$-U-factorization.

Let $\alpha \in$ irreducible, strongly irreducible, m-irreducible, unrefinably irreducible, very strongly irreducible\}. Let $a$ be a non-unit. If

$$
a=\lambda a_{1} a_{2} \cdots a_{n}\left\lceil b_{1} b_{2} \cdots b_{m}\right\rceil
$$

is a $\tau$-U-factorization, then this factorization is said to be a $\tau$-U- $\alpha$ factorization if it is a $\tau$-U-factorization and the essential divisors $b_{i}$ are $\tau-\alpha$ for $1 \leq i \leq m$.

We now define the finite factorization properties using the $\tau$-Ufactorization approach. Let $\alpha \in\{$ irreducible, strongly irreducible, $m$ irreducible, unrefinably irreducible, very strongly irreducible $\}$ and let $\beta \in\{$ associate, strongly associate, very strongly associate $\} . R$ is said to be $\tau-U$ - $\alpha$ if for all non-units $a \in R$, there is a $\tau$-U- $\alpha$-factorization of $a . \quad R$ is said to satisfy $\tau$-U-ACCP (ascending chain condition on principal ideals) if every properly ascending chain of principal ideals $\left(a_{1}\right) \subsetneq\left(a_{2}\right) \subsetneq \cdots$ such that $a_{i+1}$ is an essential divisor in some $\tau$-Ufactorization of $a_{i}$, for each $i$ terminates after finitely many principal ideals. $R$ is said to be a $\tau$-U-BFR if for all non-units $a \in R$, there is 
a bound on the number of essential divisors in any $\tau$-U-factorization of $a$.

$R$ is said to be a $\tau$-U- $\beta$-FFR if for all non-units $a \in R$, there are only finitely many $\tau$-U-factorizations up to rearrangement of the essential divisors and $\beta . \quad R$ is said to be a $\tau$-U- $\beta$-WFFR if for all non-units $a \in R$, there are only finitely many essential divisors among all $\tau$-Ufactorizations of $a$ up to $\beta . R$ is said to be a $\tau$-U- $\alpha$ - $\beta$-divisor finite (df) ring if for all non-units $a \in R$, there are only finitely many essential $\tau$ - $\alpha$ divisors up to $\beta$ in the $\tau$-U-factorizations of $a$.

$R$ is said to be a $\tau$-U- $\alpha$-HFR if $R$ is $\tau$-U- $\alpha$ and for all non-units $a \in R$, the number of essential divisors in any $\tau$-U- $\alpha$-factorization of $a$ is the same. $R$ is said to be a $\tau$-U- $\alpha-\beta$-UFR if $R$ is a $\tau$-U- $\alpha$-HFR and the essential divisors of any two $\tau$-U- $\alpha$-factorizations can be rearranged to match up to $\beta$.

The following diagram summarizes the main results from $[43$, Theorem 4.3, Theorem 4.4] where $\approx$ represents $R$ being strongly associate, $\dagger$ represents $R$ is $\tau$-U-refinable, and $\nabla$ represents $\tau$ is associate preserving and refinable:

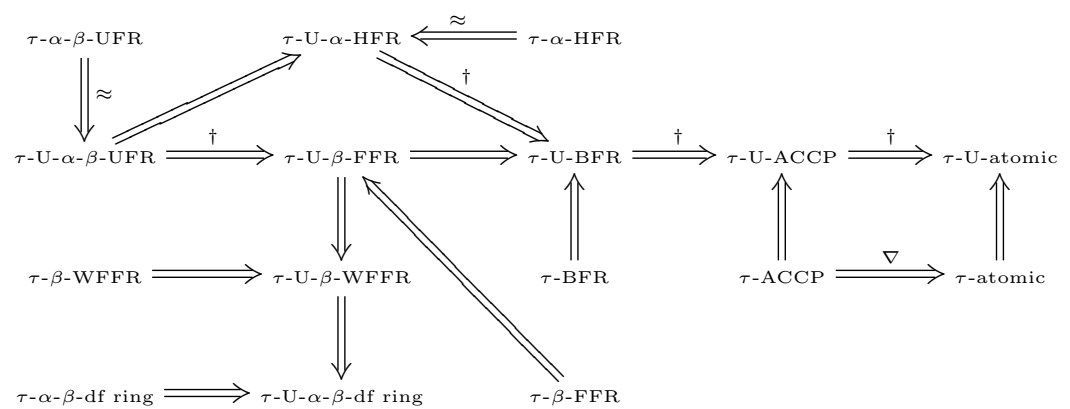

3. $\tau$-regular factorization. We will denote the set of regular elements in a ring $R$ by $\operatorname{Reg}(R)$, so $R-Z(R)=\operatorname{Reg}(R)$. We will use $\operatorname{Reg}(R)^{\#}$ to denote the regular elements which are not units. The primary benefit of looking at the factorization of the regular elements is that for regular elements, all of the associate relations coincide. That is, let $a, b \in \operatorname{Reg}(R)$, then $a \sim b$ implies $a \cong b$. Suppose $a=r b$. Neither $a$ nor $b$ can be zero, or else they could not be regular elements since we assume $R$ has an identity which is not zero. But $a \sim b$ implies there 
is an $s \in R$ such that $b=s a$. Thus $a=r b=r(s a)=(r s) a$, but $a$ is regular, so $a(1-r s)=0$ implies $r s-1=0$ or $r s=1$ and $r \in U(R)$ as desired. Another important consequence is that, for a regular element, we always have $a \cong a$. This means that for a regular, non-unit element $a \in \operatorname{Reg}(R)$, if $a$ is irreducible, then $a$ is very strongly irreducible. As a consequence, for a regular, non-unit $a \in R$ we can simply refer to it as irreducible without any ambiguity. We will soon see that this simplifies matters considerably.

3.1. $\tau$-regular factorization definitions. Let $\tau$ be a symmetric relation on $R^{\#}$. A $\tau$-factorization, $a=\lambda a_{1} \cdots a_{n}$ with $\lambda \in U(R)$, and $a_{i} \tau a_{j}$ for all $i \neq j$ is said to be a $\tau$-regular-factorization or $\tau$-rfactorization if $a \in \operatorname{Reg}(R)$. Note that $a$ is regular if and only if $a_{i}$ is regular for each $1 \leq i \leq n$.

Proposition 3.1. Let $R$ be a commutative ring with 1 and let $\tau$ be a symmetric relation on $R^{\#}$. Given $a \in \operatorname{Reg}(R)$, the following are equivalent.

(i) For any $\tau$-regular-factorization, $a=\lambda a_{1} \cdots a_{n}$, we have $a \sim a_{i}$ for some $1 \leq i \leq n$.

(ii) For any $\tau$-regular-factorization, $a=\lambda a_{1} \cdots a_{n}$, we have $a \approx a_{i}$ for some $1 \leq i \leq n$.

(iii) For any $\tau$-regular-factorization, $a=\lambda a_{1} \cdots a_{n}$, we have $a \sim a_{i}$ for all $1 \leq i \leq n$.

(iv) The only $\tau$-regular factorizations of $a$ are of the form $a=$ $\lambda\left(\lambda^{-1} a\right)$.

(v) $a \cong a$ and for any $\tau$-regular-factorization, $a=\lambda a_{1} \cdots a_{n}$, we have $a \cong a_{i}$ for some $1 \leq i \leq n$.

Proof. (v) $\Rightarrow$ (iv). Suppose $a=\lambda a_{1} \cdots a_{n}$ is a $\tau$-regular factorization with $n \geq 2$. Then by hypothesis $a \cong a_{i}$ for some $1 \leq i \leq n$. Then

$$
a=\left(\lambda a_{1} \cdots a_{i-1} \widehat{a_{i}} a_{i+1} \cdots a_{n}\right) a_{i}
$$

implies that $\left(\lambda a_{1} \cdots a_{i-1} \widehat{a_{i}} a_{i+1} \cdots a_{n}\right)$ is a unit. Hence the factorization was a trivial factorization to begin with.

(iv) $\Rightarrow$ (iii) is immediate. After noting that any divisor of a regular element must be regular and hence $\sim, \approx$ and $\cong$ coincide, it is clear that (iii) $\Rightarrow$ (ii) and (ii) $\Rightarrow$ (i). 
(i) $\Rightarrow(\mathrm{v})$. Since $a$ is regular by hypothesis, $a \cong a$ and again $\sim, \approx$ and $\cong$ coincide on any divisors of a regular element, completing the proof.

We say that a non-unit, $a \in \operatorname{Reg}(R)$ is $\tau$-r-irreducible or a $\tau$-ratom if $a$ satisfies any of the above equivalent conditions. We say $R$ is $\tau$-r-atomic if for all $a \in \operatorname{Reg}(R)^{\#}$, there is a $\tau$-r-factorization into $\tau$-r-irreducible elements. $R$ satisfies $\tau$-r-ACCP if for every chain of principal ideals generated by regular elements $\left(a_{1}\right) \subsetneq\left(a_{2}\right) \subsetneq \cdots\left(a_{i}\right) \subsetneq$ ... with $a_{i+1}$ occurring as a $\tau$-divisor in some $\tau$-r-factorization of $a_{i}$ for all $i$ becomes stationary.

$R$ is a $\tau$-r-half factorization ring (HFR) if

(1) $R$ is $\tau$-r-atomic and

(2) if $\lambda a_{1} \cdots a_{m}=\mu b_{1} \cdots b_{n}$ are two $\tau$-r-atomic $\tau$-factorizations implies that $m=n$.

$R$ is said to be a $\tau$-r-unique factorization ring (UFR) if $R$ is a $\tau$-rHFR and there is a rearrangement of any two $\tau$-r-atomic factorizations as above such that $a_{i} \sim b_{i}$ for all $1 \leq i \leq n=m$. We define the $\tau$-regular-elasticity as $\tau$-r- $\rho(R)=\sup \left\{\rho(a) \mid a \in \operatorname{Reg}(R)^{\#}\right\}$ where

$\rho(a)=\sup \left\{\frac{m}{n} \mid \lambda a=a_{1} \cdots a_{m}=\mu b_{1} \cdots b_{n}\right.$ are $\tau$-atomic-factorizations $\}$.

Then it is clear that $R$ is a $\tau$-r-HFR if and only if $R$ is $\tau$-atomic and $\tau-\mathrm{r}-\rho(R)=1$.

$R$ is said to be a $\tau$-r-bounded factorization domain (BFR) if for every $a \in \operatorname{Reg}(R)$ there exists a natural number $N_{r}(a)$ such that for all $\tau$-r-factorizations $a=\lambda a_{1} \cdots a_{n}$, we have $n \leq N_{r}(a) . R$ is said to be a $\tau$-r-irreducible-divisor-finite ring (idf ring) if each $a \in \operatorname{Reg}(R)^{\#}$ has at most a finite number of $\tau$-r-irreducible $\tau$-divisors (up to order and associates). $R$ is said to be a $\tau$-r-finite factorization ring (FFR) if for every $a \in \operatorname{Reg}(R)^{\#}$, a has only a finite number (up to order and associates) of $\tau$-factorizations. $R$ is said to be a $\tau$-r-weak finite factorization ring (WFFR) if for every $a \in \operatorname{Reg}(R)^{\#}$ there are only a finite number of $\tau$-divisors (up to order and associates).

3.2. $\tau$-regular factorization results. We begin with a useful lemma by Dickson [23]. 
Lemma 3.2 (Dickson's theorem [23]). Let $S=\mathbb{N} \times \cdots \times \mathbb{N}$ be the direct Cartesian product of $n$ copies of $\mathbb{N}$. Let $\left(a_{1}, \ldots, a_{n}\right) \leq\left(b_{1}, \ldots, b_{n}\right)$ in $S$ if and only if $a_{i} \leq b_{i}$ for $1 \leq i \leq n$. This is a partial ordering on $S$. If $\emptyset \neq X \subseteq S$, then $X$ has only finitely many minimal elements.

Proposition 3.3. Let $R$ be a commutative ring with 1 . Let $\tau$ be a symmetric relation on $R^{\#}$ with $\tau$ refinable, then the following are equivalent.

(i) $R$ is a $\tau$-r-FFR.

(ii) $R$ is a $\tau$-r-WFFR.

(iii) $R$ is a $\tau$-r-atomic $\tau$-r-idf ring.

(iv) $R$ is $\tau$-r-atomic and each $a \in \operatorname{Reg}(R)^{\#}$, a has only finitely many $\tau$-r-atomic $\tau$-factorizations up to order and associates.

(v) For all $a \in \operatorname{Reg}(R)^{\#}$, there are only finitely many $b \in \operatorname{Reg}(R)^{\#}$ up to associate such that $b$ occurs as a $\tau$-factor in $a \tau$-rfactorization of a.

(vi) For all $a \in \operatorname{Reg}(R)^{\#}$, (a) is contained in only finitely many principal ideals $(b)$ where $b \in \operatorname{Reg}(R)^{\#}$ such that $b$ occurs as a $\tau$-factor in a $\tau$-r-factorization of $a$.

(vii) For all $a \in \operatorname{Reg}(R)^{\#}$, there are only finitely many $b \in \operatorname{Reg}(R)^{\#}$ up to associate such that $\left.b\right|_{\tau}$ a.

(viii) For all $a \in \operatorname{Reg}(R)^{\#}$, (a) is contained in only finitely many principal ideals $(b)$ where $b \in \operatorname{Reg}(R)^{\#}$ such that $\left.b\right|_{\tau} a$.

Proof. (i) $\Rightarrow$ (ii). Letting $R$ be a $\tau$-r-FFR and $a \in \operatorname{Reg}(R)^{\#}$, then there are only a finite number of $\tau$-factorizations (up to order and associate), each of finite length. Hence, since every $\tau$-divisor of $a$ must be among these up to associate, $R$ is a $\tau$-r-WFFR.

(ii) $\Rightarrow$ (iii). Let $R$ be a $\tau$-r-WFFR and $a \in \operatorname{Reg}(R)^{\#}$. If $a$ has a finite number of $\tau$-divisors, then certainly it has a finite number of irreducible $\tau$-divisors, so it suffices to show $a$ has a $\tau$-r-atomic factorization. We instead show the stronger condition, that $R$ satisfies $\tau$-r-ACCP, that is, any chain of principal ideals generated by regular elements

$$
\left(a_{0}\right) \subsetneq\left(a_{1}\right) \subsetneq \cdots \subsetneq\left(a_{i}\right) \subsetneq \cdots
$$

with $a_{i+1}$ occurring as a $\tau$-factor in a $\tau$-r-factorization of $a_{i}$ and $a_{i} \in \operatorname{Reg}(R)^{\#}$ for all $i$ comes to a halt. Suppose there is an infinite chain, but then each $a_{i}$ is a $\tau$-divisor of $a_{0}$ and none of them is associate 
since each containment is proper. Therefore we would have an infinite number of non-associate $\tau$-r-divisors contradicting the fact that $R$ is a $\tau$-r-WFFR (note, here we emphasize that $\tau$ is refinable to ensure that at each step we retain a $\tau$-factorization).

(iii) $\Rightarrow$ (i). We use Dickson's theorem and modify the proof of [2, Theorem 4]. Let $R$ be a $\tau$-r-atomic $\tau$-r-idf ring and $a \in \operatorname{Reg}(R)^{\#}$. Let $a_{1}, \ldots, a_{n}$ be the $\tau$-r-irreducible $\tau$-factors of $a$ representatives up to associate. In particular, they are all regular elements of $R$ since they divide a regular element. Because $\tau$ is refinable and $R$ is $\tau$-r-atomic, any $\tau$-r-factorization of $a=\lambda b_{1} \cdots b_{n}$ can be $\tau$-refined into a $\tau$-r-atomic factorization, it suffices to show that there are a finite number of $\tau$-ratomic factorizations of $a$ up to rearrangement and associate.

Given any $\tau$-r-atomic factorization of $a$, since $\tau$ is refinable, we can replace each $\tau$-atomic factor with one from the list $a_{1}, \ldots, a_{n}$ and collect all the unit multiples in the front with the $\lambda \in U(R)$. Thus, given any $\tau$-r-atomic factorization of $a$, we let $e_{i}$ represent the number of not necessarily distinct $\tau$-r-factors in the $\tau$-factorization which are associates of $a_{i}$. We then could group the terms together based on the associates of the $n$ given $\tau$-r-atomic divisors of $a$ as follows

$$
a=\lambda a_{1}^{e_{1}} \cdot a_{2}^{e_{2}} \cdots a_{n}^{e_{n}} .
$$

Then $e_{i}$ is a non-negative integer for each $1 \leq i \leq n$ representing the number of times an associate of $a_{i}$ appears as a factor in the given $\tau$-ratomic factorization of $a$. Consider the set $X=\left\{\left(e_{1}, \ldots, e_{n}\right)\right\}$, which stem from the $\tau$-r-atomic factorizations in this way. Since $R$ is $\tau$-ratomic, $X \neq \emptyset$. We use the partial ordering provided by Lemma 3.2. Let $\left(e_{i}\right),\left(f_{i}\right) \in X$ be such that $\left(e_{i}\right) \leq\left(f_{i}\right)$. If $\left(e_{i}\right) \neq\left(f_{i}\right)$, then there is some $e_{i}<f_{i}, 1 \leq i \leq n$. Without loss of generality, we suppose it is $e_{1}<f_{1}$. This would imply that

$$
(a)=\left(a_{1}^{f_{1}} \cdot a_{2}^{f_{2}} \cdots a_{n}^{f_{n}}\right) \subsetneq\left(a_{1}^{e_{1}} \cdot a_{2}^{f_{2}} \cdots a_{n}^{f_{n}}\right)=\left(a_{1}^{e_{1}} a_{2}^{e_{2}} \cdots a_{n}^{e_{n}}\right)=(a) .
$$

This is a contradiction, so each element of $X$ is in fact minimal in $X$. By Dickson's theorem, the set of minimal elements is finite. This means $X$ itself must be finite. Hence there are only a finite number of $\tau$-r-atomic factorizations of $a$ up to rearrangement and associates. Thus $R$ is a $\tau$-r-FFR as desired.

(i) $\Rightarrow$ (iv). This is clear as we have already seen that a $\tau$-r-FFR is $\tau$ r-atomic and a $\tau$-r-atomic factorization is certainly a $\tau$-r-factorization, 
so there must be a finite number of $\tau$-r-atomic factorizations up to order and associate for every $a \in \operatorname{Reg}(R)^{\#}$.

(iv) $\Rightarrow$ (iii). Letting $a \in \operatorname{Reg}(R)^{\#}$, then there are a finite number of $\tau$-r-atomic factorizations, each of which has a finite number of $\tau$-ratomic factors. Therefore the collection of $\tau$-r-atomic divisors is finite, so $R$ is a $\tau$-r-atomic $\tau$-r-idf ring.

(v) and (vi) are restatements of (ii) and their equivalence is immediate. Furthermore, (v) and (vii) (respectively (vi) and (viii)) are seen to be equivalent after noting that for $b \in \operatorname{Reg}(R),\left.a\right|_{\tau} b$ implies there is some $\tau$-factorization $b=\lambda a a_{1} \cdots a_{n}$, but since $b$ is regular and the set of regular elements is saturated, every $\tau$-factor must be regular so this is really a $\tau$-factorization.

Theorem 3.4. Let $R$ be a commutative ring with 1 , with $\tau$ a symmetric relation on $R^{\#}$. We have the following:

(i) $R$ is a $\tau$-r-UFR implies $R$ is a $\tau$-r-HFR.

(ii) For $\tau$ refinable, $R$ is a $\tau$-r-HFR implies $R$ is a $\tau$-r-BFR.

(iii) For $\tau$ refinable, $R$ is a $\tau$-r-UFR implies $R$ is a $\tau$-r-FFR.

(iv) $R$ is a $\tau$-r-FFR implies $R$ is a $\tau$-r-BFR.

(v) For $\tau$ refinable, $R$ is a $\tau$-r-BFR implies $R$ satisfies $\tau$-r-ACCP.

(vi) For $\tau$ refinable, $R$ satisfies $\tau$-r-ACCP implies $R$ is $\tau$-r-atomic.

Proof.

(i) This is immediate from the definition.

(ii) Let $R$ be a $\tau$-r-HFR. Suppose $a=\lambda a_{1} \cdots a_{n}$ is a $\tau$-r-atomic factorization. We claim $N_{r}(a)=n$. Let $a=\mu b_{1} \cdots b_{m}$ be a $\tau$ r-factorization of $a$. Since $R$ is $\tau$-r-atomic, we can find $\tau$-r-atomic factorizations for $b_{i}, 1 \leq i \leq m$. We have assumed $\tau$ to be refinable, so we can replace each $b_{i}$ with the corresponding $\tau$-r-atomic factorization and collect the units in front of the factorization and retain a $\tau$-rfactorization which is $\tau$-atomic and thus must have length $n$. The refinement process can only increase the length of the factorization, so the length of the original factorization is no longer than $n$, proving the claim.

(iii) We show for $\tau$-refinable, $R$ a $\tau$-r-UFR, $R$ is a $\tau$-r-atomic $\tau$-r-idfring which has been shown in Theorem 3.3 to be equivalent to being a 
$\tau$-r-FFR. Since $R$ is a $\tau$-r-UFR, we get $\tau$-r-atomic for free. Furthermore, any $\tau$-atomic factorization of $a \in \operatorname{Reg}(R)^{\#}$ has the same length, say $n$, and can be reordered so that the associates match up. This tells us there are precisely $n \tau$-irreducible divisors of $a$ up to associate; hence, $R$ is a $\tau$-r-idf-ring.

(iv) Suppose $R$ is a $\tau$-r-FFR; by definition, we know $R$ is $\tau$-r-atomic. Now, let $a \in \operatorname{Reg}(R)^{\#}$ and let $S$ be the finite set of all $\tau$-atomic factors of $a$. Set $N(a)=|S|$. Letting $a=\lambda a_{1} \cdots a_{n}$ be a $\tau$-atomic factorization of $a$, then $a_{i} \in S$ for all $i$, but then $\left\{a_{i}\right\}_{i=1}^{n} \subseteq S$ and hence is finite and $n \leq N(a)=|S|$ as desired. So $R$ is a $\tau-r$-BFR.

(v) Let $R$ be a $\tau$-r-BFR, and suppose for a moment that $R$ does not satisfy $\tau$-r-ACCP. There must exist an infinite sequence $\left\{a_{i}\right\}_{i=1}^{\infty} \subseteq$ $\operatorname{Reg}(R)^{\#}$ such that $\left.a_{n+1}\right|_{\tau} a_{n}$, but $a_{n+1} \nsim a_{n}$ for all $n \geq 1$. Let $a_{n}=\lambda_{n+1} r_{n+11} \cdots r_{n+1 s_{n+1}} a_{n+1}$ be a $\tau$-factorization of $a_{n}$ for all $n \geq 1$. But then we have

$$
a_{1}=\lambda_{2} r_{2_{1}} \cdots r_{2_{s_{2}}} a_{2}=\lambda_{2} r_{2_{1}} \cdots r_{2_{s_{2}}} \lambda_{3} r_{3_{1}} \cdots r_{3_{s_{3}}} a_{3}=\cdots
$$

is a $\tau$-factorization (note we use $\tau$ refinable here). This shows that we can find arbitrarily large $\tau$-factorizations of $a_{1}$, which contradicts the fact the $R$ is a $\tau$-r-BFR.

(vi) Let $R$ satisfy $\tau$-r-ACCP, but suppose that $R$ is not $\tau$-r-atomic. Then there exists $a \in \operatorname{Reg}(R)^{\#}$ with no $\tau$-factorization into $\tau$-atoms. $a$ itself cannot be a $\tau$-atom, so say $a=\lambda a_{1} \cdots a_{n}$ is a $\tau$-factorization with $n>1$. Now again, some $a_{i}$ must not be a product of $\tau$-atoms, or with $\tau$ refinable, we could find a $\tau$-atomic factorization, say $a_{1}$. Then $\left.a_{1}\right|_{\tau} a$ and $a_{1} \neq a$ produce $b_{1}=a_{1}$. Then $a_{1}$ must have a $\tau$-factorization $a_{1}=\lambda_{2} a_{2_{1}} \cdots a_{2_{n_{2}}}$ where $n_{2}>1$. Again, one of the $\tau$-factors, say $a_{2_{1}}$ cannot be a $\tau$-product of $\tau$-atoms. Here $\left.a_{2_{1}}\right|_{\tau} a_{1}=b_{1}$ and $a_{2_{1}} \nsim a_{1}$. Put $b_{2}=a_{2_{1}}$. Continuing in this fashion, we obtain a sequence $\left\{b_{i}\right\}_{i=1}^{\infty}$ of elements of $\operatorname{Reg}(R)^{\#}$ such that $\left.b_{n+1}\right|_{\tau} b_{n}$ but $b_{n+1} \nsim b_{n}$ for every $n \geq 1$. This contradicts $R$ satisfying $\tau$-r-ACCP.

The following diagram summarizes our result where $\nabla$ represents $\tau$ refinable.

4. $\tau_{\text {reg-factorizations. In this section, we study another approach }}$ which could have been used to extend $\tau$-factorization to commutative rings with zero-divisors using regular factorizations. In Section 3, we 


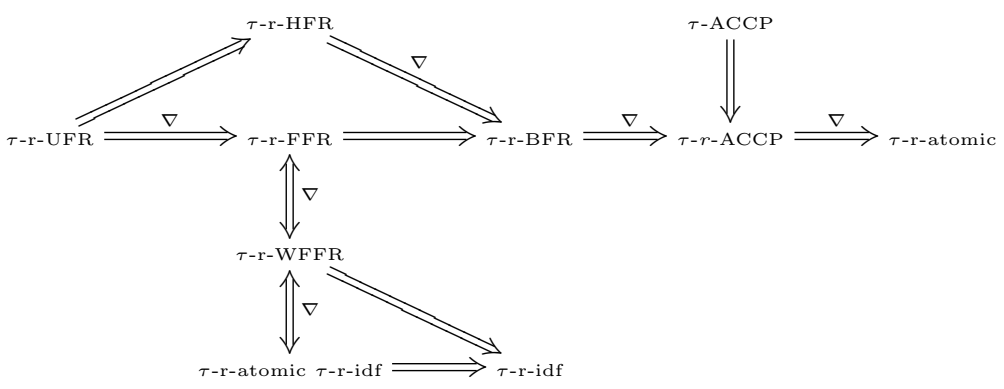

decided to consider only factorizations of the regular elements. In other words, we chose to restrict the elements we attempt to factor to the regular elements of a commutative ring $R$. We could have instead chosen to restrict the relation $\tau$ itself. This gives us the benefit of not completely ignoring a possible large number of zero-divisors in the ring $R$, but at the cost of choosing a less natural relation $\tau$. Moreover, it allows us to use much of the work done previously in [42] by just picking a different $\tau$ and keeping all of the original definitions the same. It turns out that, in many ways, either choice is fine and we end up at the same place anyway. Studying this will be the motivation of this section.

Let $R$ be a commutative ring with 1 and $\tau$ a symmetric relation on $R^{\#}$. Then we define a new relation

$$
\tau_{\text {reg }}:=\tau \cap(\operatorname{Reg}(R) \times \operatorname{Reg}(R)) .
$$

We may now pursue the $\tau$-factorizations using the approach from [42] and look at factoring all the non-units in $R$ instead of just the regular elements. There is certainly a very close relationship between

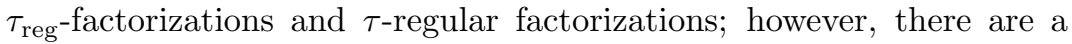
few subtle differences that cause some problems, especially with the

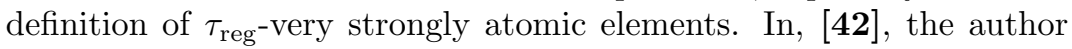
insisted that part of $a$ being $\tau$-very strongly atomic was that $a \cong a$.

The fact that the very strongly associate relation need not be reflexive is the main reason there is not a perfect correspondence between the two approaches. We will see that $\tau_{\text {reg-factorizations are }}$ simply very poorly behaved when it comes to $\tau_{\text {reg-very strong atoms }}$ and rearrangement up to very strong associates. On the bright side, the $\tau$-unrefinably irreducible element introduced in [44] will also behave quite nicely here. 
Of course, any non-trivial idempotent element, $e$, is a zero-divisor since $e(e-1)=0$. Furthermore, since $e=e^{2}=e \cdot e$, with $e$ not a unit, we see that $e \neq e$. This means that $e$ is not very strongly atomic for any non-trivial idempotent element. On the other hand, since every

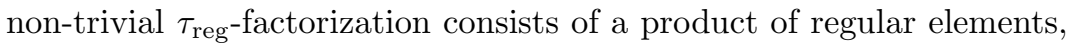

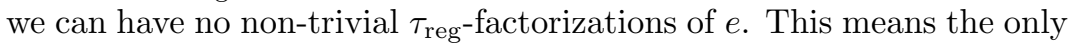

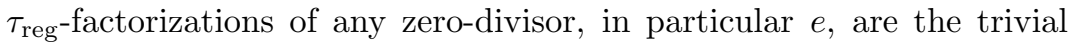
factorizations. Unfortunately, in the case of a non-trivial idempotent, $e$, this means $e$ is not a $\tau$-very strong atom and will never have a

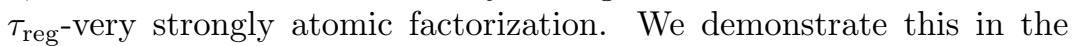
following example.

Example 4.1. Let $K$ be an infinite field,

$$
R=K \times K \quad \text { with } \tau=R^{\#} \times R^{\#} .
$$

We consider the element $(1,0) \in Z(R)$. This ring has only elements which are strongly associate to idempotent elements and units. So the set of non-unit regular elements is empty and our ring is vacuously a $\tau$ r-UFR. On the other hand, we have $(1,0)=\left(\mu^{-1}, 1\right)(\mu, 0)$, for any unit $\mu \in K^{*}$, which is the only type of $\tau_{\text {reg-factorization of }}(1,0)$. Yet, none of these are $\tau_{\text {reg-very strongly atomic factorizations. The problem is }}$ that $(\mu, 0) \nRightarrow(\mu, 0)$ since we have $(\mu, 0)=(1,0)(\mu, 0)$ and $(1,0)$ is not a unit. This shows we can have a $\tau$-r-UFR which is not even $\tau_{\text {reg-atomic. }}$ Moreover, each of these factorizations is non-very strongly associate.

Let $\mu, \lambda \in K^{*}$. Then $(1,0)=\left(\mu^{-1}, 1\right)(\mu, 0)=\left(\lambda^{-1}, 1\right)(\lambda, 0)$ are two

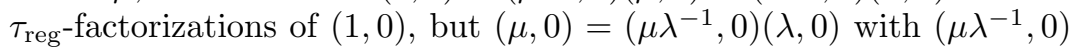
not a unit shows $(\mu, 0) \nsucc(\lambda, 0)$. Since $K$ is infinite, there are infinitely

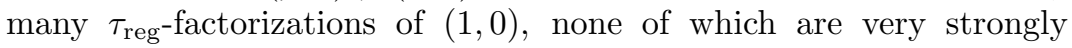
associate.

This leads us to the following results.

Lemma 4.2. Let $R$ be a commutative ring with 1 , and let $\tau$ be a symmetric relation on $R^{\#}$. Let

$$
\tau_{\text {reg }}:=\tau \cap(\operatorname{Reg}(R) \times \operatorname{Reg}(R)) .
$$

The collection of non-trivial $\tau$-regular-factorizations and non-trivial $\tau_{\text {reg-factorizations coincide. }}$ 
Proof. Let $a=\lambda a_{1} \cdots a_{n}$ be a non-trivial $\tau$-regular factorization. Then $a \in \operatorname{Reg}(R)$ by definition of $\tau$-regular factorization, and $a_{i} \tau a_{j}$ for all $i \neq j$. Since $a$ is regular, and the set of regular elements is saturated, we have $a_{i} \mid a \in \operatorname{Reg}(R)$ for each $1 \leq i \leq n$, and we know that $a_{i} \in \operatorname{Reg}(R)$ for each $1 \leq i \leq n$. This means $a_{i} \tau_{\text {reg }} a_{j}$ for each $i \neq j$. Thus, $a=\lambda a_{1} \cdots a_{n}$ is a $\tau_{\text {reg-factorization. }}$

Conversely, suppose $a=\lambda a_{1} \cdots a_{n}$ is a non-trivial $\tau_{\text {reg-factorization. }}$ Then $a_{i} \tau_{\text {reg }} a_{j}$ for each $i \neq j$. This means $a_{i} \tau a_{j}$ and $a_{i}, a_{j} \in \operatorname{Reg}(R)$. In particular, since $n \geq 2$, we can conclude that $a_{1} a_{2} \cdots a_{n}$ is a product of regular elements, so $a \in \operatorname{Reg}(R)$. This means $a=\lambda a_{1} \cdots a_{n}$ is a $\tau$-regular-factorization.

Theorem 4.3. Let $R$ be a commutative ring with 1 , and let $\tau$ be a symmetric relation on $R^{\#}$. Let

$$
\tau_{\text {reg }}:=\tau \cap(\operatorname{Reg}(R) \times \operatorname{Reg}(R)) .
$$

For $a \in \operatorname{Reg}(R)$, the following are equivalent:

(i) $a$ is a $\tau$-regular-atom.

(ii) $a$ is a $\tau_{\text {reg-atom. }}$

(iii) $a$ is a $\tau_{\text {reg-strong atom. }}$

(iv) $a$ is a $\tau_{\text {reg-m-atom. }}$

(v) a is a $\tau_{\text {reg-unrefinable atom. }}$

(vi) $a$ is a $\tau_{\text {reg-very strong atom. }}$

Proof. When we consider Theorem 2.2, it suffices to show that (ii) $\Rightarrow(\mathrm{vi})$ and then we show that (i) $\Leftrightarrow(\mathrm{v})$. Let $a \in \operatorname{Reg}(R)$ be a $\tau_{\text {reg }^{-}}$ atom. Since $a \in \operatorname{Reg}(R)$, we have $a \cong a$ since $a=r a$ implies $r=1$. Furthermore, if $a=\lambda a_{1} \cdots a_{n}$ is a $\tau_{\text {reg-factorization of } a \text {, then } a \sim a_{i}}$ for some $1 \leq i \leq n$. Since $a \in \operatorname{Reg}(R), a \cong a_{i}$ and we have shown that $a$ is a $\tau_{\text {reg-very strongly atom. }}$

(i) $\Leftrightarrow$ (v). In light of Lemma 4.2, $a$ has a non-trivial $\tau$-regular factorization if and only if $a$ has a non-trivial $\tau_{\text {reg-factorization. }}$

Corollary 4.4. Let $R$ be a commutative ring with 1 , and let $\tau$ be a symmetric relation on $R^{\#}$. Let

$$
\tau_{\text {reg }}:=\tau \cap(\operatorname{Reg}(R) \times \operatorname{Reg}(R)) .
$$


Let

$\alpha \in\{$ atomic, strongly atomic, m-atomic, unrefinably atomic $\}$.

Letting $a \in \operatorname{Reg}(R)$ be a non-unit, then $a=\lambda a_{1} \cdots a_{n}$ is a $\tau_{\text {reg- }}$ $\alpha$-factorization if and only if $a=\lambda a_{1} \cdots a_{n}$ is a $\tau$-regular-atomic factorization.

Proof. This is immediate from what we have shown in Theorem 4.3.

Theorem 4.5. Let $R$ be a commutative ring with 1 , and let $\tau$ be a symmetric relation on $R^{\#}$. Let

$$
\tau_{\text {reg }}:=\tau \cap(\operatorname{Reg}(R) \times \operatorname{Reg}(R)) .
$$

If $a \in Z(R)$, then the following hold:

(i) a is a $\tau_{\text {reg-atom. }}$

(ii) a is a $\tau_{\text {reg-strong atom. }}$

(iii) $a$ is a $\tau_{\text {reg-m-atom. }}$

(iv) a is a $\tau_{\text {reg-unrefinable atom. }}$

Proof. By Theorem 2.2, it suffices to show that, for $a \in Z(R)$,

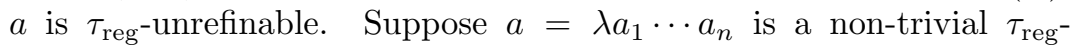
factorization. This implies $n \geq 2$, and therefore $a_{i} \tau_{\text {reg }} a_{j}$ for each $i \neq j$. In particular, $a_{i} \in \operatorname{Reg}(R)$ for all $1 \leq i \leq n$. This means $a$ is a product of regular elements and is therefore regular, and hence not a zero-divisor, a contradiction. Thus there are only non-trivial $\tau_{\text {reg- }}$

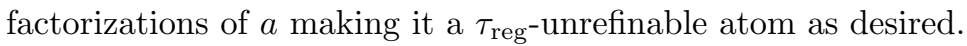

Theorem 4.6. Let $R$ be a commutative ring with 1 , and let $\tau$ be a symmetric relation on $R^{\#}$. Let

$$
\tau_{\text {reg }}:=\tau \cap(\operatorname{Reg}(R) \times \operatorname{Reg}(R)) .
$$

The following are equivalent:

(i) $R$ is $\tau$-regular-atomic.

(ii) $R$ is a $\tau_{\text {reg-atomic. }}$

(iii) $R$ is a $\tau_{\text {reg-strongly atomic. }}$

(iv) $R$ is a $\tau_{\text {reg-m-atomic. }}$

(v) $R$ is $\tau_{\text {reg-unrefinably atomic. }}$ 
Proof. Let $a$ be a non-unit in $R$. Then $a \in Z(R)$ or $a \in \operatorname{Reg}(R)$. If $a \in Z(R)$, we apply Theorem 4.5 to see that $a$ itself is $\tau_{\text {reg-atomic, }}$ $\tau_{\text {reg-strongly atomic, }} \tau_{\text {reg-m-atomic, }}$, and $\tau_{\text {reg- }}$-unrefinably atomic; and $a=1 \cdot a$ is a $\tau_{\text {reg-atomic, }} \tau_{\text {reg-strongly atomic, }} \tau_{\text {reg-m-atomic, and }}$

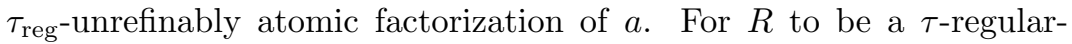
atomic ring, we need only check the regular elements for $\tau$-regular atomic factorizations. If $a \in \operatorname{Reg}(R)$, we apply Corollary 4.4 to see that $a$ has a $\tau$-regular-atomic factorization if and only if $a$ has a $\tau_{\text {reg-atomic }}$ (respectively $\tau_{\text {reg }}$-strongly atomic, $\tau_{\text {reg-m}}$-m-atomic, $\tau_{\text {reg- }}{ }^{-}$ unrefinably atomic) factorization. This completes the equivalence since we have checked both the zero-divisors as well as the regular elements.

Lemma 4.7. Let $R$ be a commutative ring with 1 , and let $\tau$ be a symmetric relation on $R^{\#}$. Let $a=\lambda\left(\lambda^{-1} a\right)=\mu\left(\mu^{-1} a\right)$ be two trivial factorizations of $a$. Then we have the following:

(i) $\lambda^{-1} a$ and $\mu^{-1} a$ are associates.

(ii) $\lambda^{-1} a$ and $\mu^{-1} a$ are strong associates.

Proof. $\left(\mu^{-1} \lambda\right)\left(\lambda^{-1} a\right)=\mu^{-1} a$ with $\left(\mu^{-1} \lambda\right) \in U(R)$ proves $\lambda^{-1} a \approx$ $\mu^{-1} a$. If $\lambda^{-1} a \approx \mu^{-1} a$, then $\lambda^{-1} a \sim \mu^{-1} a$. This proves both (ii) and (i).

Remark 1 . Given the above situation, $\lambda^{-1} a$ and $\mu^{-1} a$ need not be very strong associates. For instance $R=\mathbb{R} \times \mathbb{R}$,

$$
(1,0)=(1,1)(1,0)=(-1,-1)(-1,0),
$$

yet $(1,0) \varsubsetneqq(-1,0)$.

Theorem 4.8. Let $R$ be a commutative ring with 1 , and let $\tau$ be a symmetric relation on $R^{\#}$. Let

$$
\tau_{\text {reg }}:=\tau \cap(\operatorname{Reg}(R) \times \operatorname{Reg}(R)) .
$$

Let

$\alpha \in\{$ atomic, strongly atomic, m-atomic, unrefinably atomic $\}$ 
and

$\beta \in\{$ associate, strongly associate $\}$.

Then we have the following:

(i) $R$ satisfies $\tau$-regular-ACCP if and only if $R$ satisfies $\tau_{\text {reg-ACCP. }}$

(ii) $R$ is a $\tau$-regular-UFR if and only if $R$ is a $\tau_{\text {reg }}-\alpha-\beta$-UFR.

(iii) $R$ is a $\tau$-regular-HFR if and only if $R$ is a $\tau_{\text {reg- }}-\alpha$-HFR.

(iv) $R$ is a $\tau$-regular-BFR if and only if $R$ is a $\tau_{\text {reg-BFR. }}$.

(v) $R$ is a $\tau$-regular-idf ring if and only if $R$ is a $\tau_{\text {reg- }}-\alpha-\beta$-df ring.

(vi) $R$ is a $\tau$-regular-atomic $\tau$-regular-idf ring if and only if $R$ is a $\tau_{\text {reg }}-\alpha, \tau_{\text {reg }}-\alpha-\beta$-df ring.

(vii) $R$ is a $\tau$-regular-WFFR if and only if $R$ is a $\tau_{\text {reg- }}-\beta$-WFFR.

(viii) $R$ is a $\tau$-regular-FFR if and only if $R$ is a $\tau_{\text {reg }}-\beta-F F R$.

If $\tau$ is refinable, then $(\mathrm{vi}) \Leftrightarrow(\mathrm{vii}) \Leftrightarrow$ (viii).

Proof.

(i) The statement that $(a) \subsetneq\left(a_{1}\right)$ with $\left.a_{1}\right|_{\tau} a$ implies that $a=\lambda a_{1} a_{2} \cdots a_{n}$. We notice here that $n \geq 2$ or else we would have $a=\lambda a_{1}$ or $a \approx a_{1}$ which implies $(a)=\left(a_{1}\right)$, a contradiction. So these properly ascending chains yield non-trivial factorizations at each step. Thus any properly ascending chain of principal ideals

$$
\left(a_{1}\right) \subsetneq\left(a_{2}\right) \subsetneq\left(a_{3}\right) \subsetneq \cdots
$$

such that $\left.a_{i+1}\right|_{\tau_{\text {reg }}} a_{i}$ yields a $\tau$-regular factorization of $a_{i}$ with $a_{i+1}$ as a $\tau$-regular factor. Conversely, any ascending chain as in (4.1) with $a_{i}$ regular for all $i$ and $a_{i+1}$ occurring as a $\tau$-factor in some $\tau$-regular

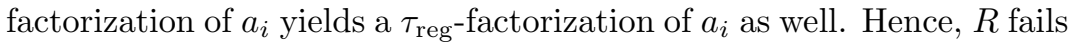
to satisfy $\tau$-regular ACCP if and only if $R$ fails to satisfy $\tau_{\text {reg-ACCP, }}$ and the proof is complete.

(ii) We know from Theorem 4.6 that $R$ is $\tau$-regular- $\alpha$ if and only if $R$ is $\tau_{\text {reg }}-\alpha$. Let $a \in R$ be a non-unit. If $a \in Z(R)$, we know from Theorem 4.5 that $a$ is $\tau_{\text {reg }}-\alpha$. Furthermore, any trivial $\tau_{\text {reg-factorization }}$ of $a$ is unique up to $\beta$ by Lemma 4.7. For $R$ to be a $\tau$-regular UFR, we need only check the regular elements. Let $a \in \operatorname{Reg}(R)$. We know from Corollary 4.4, for regular elements, $\tau$-atomic and $\tau_{\text {reg }}-\alpha$ factorizations of $a$ coincide, so the uniqueness up to rearrangement and $\beta$ is immediate. 
(iii) By Theorem 4.6, $R$ is $\tau$-regular- $\alpha$ if and only if $R$ is $\tau_{\text {reg }}-\alpha$. If $a \in Z(R)$, then $a$ is $\tau_{\text {reg }}-\alpha$ and has only trivial $\tau_{\text {reg-factorizations, }}$ each of which has length 1 . For $a \in \operatorname{Reg}(R), \tau$-atomic and $\tau_{\text {reg- }}-\alpha-$ factorizations of $a$ coincide by Corollary 4.4, and the equivalence is clear.

(iv) For $a \in Z(R)$, all $\tau_{\text {reg-factorizations are trivial and have length } 1 .}$ By Lemma 4.2 , the set of non-trivial $\tau$-regular factorizations and $\tau_{\text {reg }^{-}}$ factorizations coincide, and the equivalence is apparent.

(v) If $a \in Z(R), a$ itself is $\tau_{\text {reg- }}-\alpha$, and there is precisely one unique $\tau_{\text {reg }}-\alpha$-divisor of $a$ up to $\beta$ since all trivial $\tau_{\text {reg }}$-factorizations are $\beta$ from Lemma 4.7. If $a \in \operatorname{Reg}(R)$, then the set of $\tau$-regular atomic divisors and $\tau_{\text {reg }}-\alpha$-divisors of $a$ are all regular and hence coincide by Theorem 4.3. So the equivalence is clear.

(vi) This is simply (v) plus Theorem 4.6.

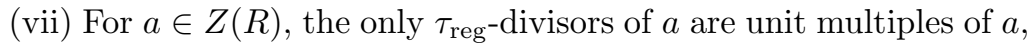

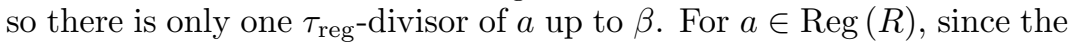
set of $\tau$-regular factorizations and the set of $\tau_{\text {reg-factorizations of } a \text { are }}$ the same, the set of $\tau_{\text {reg-divisors and } \tau \text {-regular divisors coincide and are }}$ regular, so the associate relations also coincide. Thus the equivalence follows.

(viii) For $a \in Z(R)$, the only $\tau_{\text {reg-factorizations of } a \text { are of the form }}$

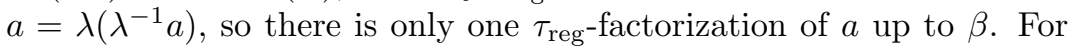
$a \in \operatorname{Reg}(R)$, the set of $\tau$-regular factorizations and the set of $\tau_{\text {reg- }}$ factorizations of $a$ are the same. Moreover, the set of $\tau_{\text {reg-factors and }}$ $\tau$-regular factors coincide and are regular; hence, the associate relations also coincide. Thus, the equivalence follows.

5. Relationship with other finite factorization properties. In this final section, we would like to demonstrate where the rings satisfying the properties in the present article fit in with the various finite factorization properties already existing in the literature, that is, we would like to compare the $\tau$-regular and $\tau_{\text {reg-finite factorization prop- }}$ erties with the regular factorization from [10], the $\tau$-finite factorization properties defined originally in [42] as well as the $\tau$-U-finite factorization properties defined in [43]. As a note to the reader, many of these terms were defined in Section 2. 
The following theorem demonstrates that the $\tau$-finite factorization properties defined in [42] are stronger than the ones in the present article.

Theorem 5.1. Let $R$ be a commutative ring with 1 , and let $\tau$ be a symmetric relation on $R^{\#}$. Let $\alpha \in\{$ atomic, strongly atomic, m-atomic, unrefinably atomic, very strongly atomic $\}, \beta \in\{$ associate, strongly associate, very strongly associate $\}$. Then we have the following:

(i) If $R$ is a $\tau$ - $\alpha-\beta$-UFR, then $R$ is a $\tau$-r-UFR.

(ii) If $R$ is a $\tau$ - $\alpha$-HFR, then $R$ is a $\tau$-r-HFR.

(iii) If $R$ is a $\tau$ - $\beta$-FFR, then $R$ is a $\tau$ - $r$-FFR.

(iv) If $R$ is a $\tau$ - $\beta$-WFFR, then $R$ is a $\tau$-r-WFFR.

(v) If $R$ is a $\tau-\beta$ - $\alpha$-df ring, then $R$ is a $\tau$-r-idf ring.

(vi) If $R$ is a $\tau$-BFR, then $R$ is a $\tau$-r-BFR.

(vii) If $R$ satisfies $\tau$-ACCP, then $R$ satisfies $\tau$-r-ACCP.

(viii) If $R$ is $\tau-\alpha$, then $R$ is $\tau$-r-atomic.

This yields the following diagram where $\nabla$ represents $\tau$ refinable.

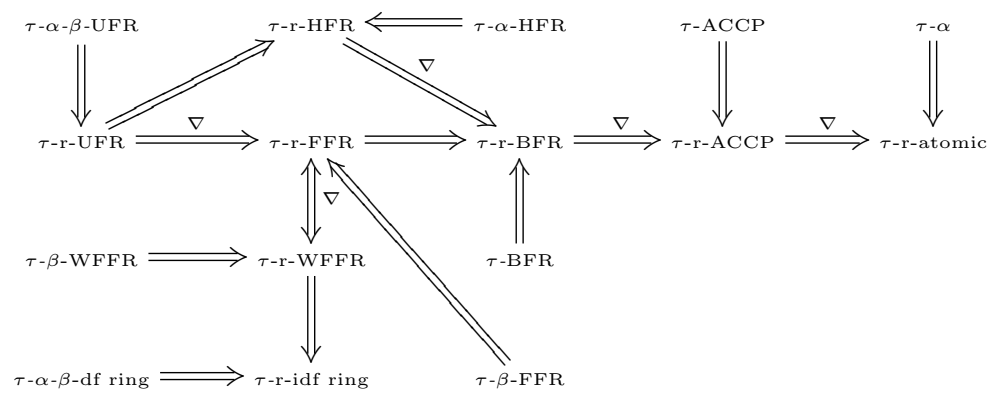

Proof.

(viii) Let $a \in \operatorname{Reg}(R)$. Since $R$ is a $\tau$ - $\alpha$, there is a $\tau$ - $\alpha$-factorization of the form $a=\lambda a_{1} \cdots a_{n}$. Since $a \in \operatorname{Reg}(R), a_{i} \in \operatorname{Reg}(R)$ for all $i$, by Proposition 3.1, each of these factorizations is a $\tau$-r-atomic factorization of $a$, showing $R$ is $\tau$-r-atomic.

(ii) (respectively (i)). Let $a$ be a regular non-unit element. We have just seen that $R$ is $\tau$-r-atomic. Given two $\tau$-r-atomic factorizations, $a=\lambda a_{1} \cdots a_{n}=\mu b_{1} \cdots b_{m}$, there are also two $\tau$ - $\alpha$-factorizations. By assumption, we have $m=n$ (respectively and there is a rearrangement 
so that $a_{i} \sim b_{i}$ for each $\left.1 \leq i \leq n\right)$. This proves $R$ is a $\tau$-r-HFR (respectively $\tau$-r-UFR).

(iii)-(vi). Let $a \in \operatorname{Reg}(R)$. For a regular element $a$, the set of $\tau$-rfactorizations and $\tau$-factorizations are identical, proving (iii) and (vi). Similarly, since every divisor of a regular element is regular, the set of regular $\tau$-divisors is the same as the set of $\tau$-divisors, proving (iv). As in 3.1, we know that the set of $\tau$ - $\alpha$-divisors is the same as the set of $\tau$-r-atoms, proving $(\mathrm{v})$.

(vii) Suppose $\left(a_{1}\right) \subsetneq\left(a_{2}\right) \subsetneq \cdots$ is a chain of regular principal ideals such that $\left.a_{i+1}\right|_{\tau} a_{i}$. Then, since $R$ satisfies $\tau$-ACCP, it must become stationary, proving (vii).

The following gives us a comparison of the regular factorization rings defined in [10] with the rings defined in the current article.

Theorem 5.2. Let $R$ be a commutative ring with 1 and $\tau \subset \operatorname{Reg}(R)^{\#} \times$ $\operatorname{Reg}(R)^{\# \text { : }}$

(i) $R$ an r-BFR implies $R$ is a $\tau$-r-BFR,

(ii) $R$ an r-FFR implies $R$ is a $\tau$-r-FFR,

(iii) $R$ an $\mathrm{r}$-WFFR implies $R$ is a $\tau$-r-WFFR,

(iv) $R$ satisfies $r$-ACCP implies $R$ satisfies $\tau$-r-ACCP.

\section{Proof.}

(i) Let $R$ be an r-BFR, but suppose $R$ is not a $\tau$-r-BFR. Then there exists a regular element $a \in \operatorname{Reg}(R)^{\#}$ with $\tau$-factorizations of arbitrarily long length, but any $\tau$-factorization is certainly a factorization into regular elements, so this would contradict the fact that $R$ is an r-BFR.

(ii) Let $R$ be an r-FFR, but suppose that $R$ is not a $\tau$-r-FFR. We then have a regular element $a \in \operatorname{Reg}(R)^{\#}$ that has an infinite number of $\tau$-r-factorizations up to rearrangement and associate, but again each of these is also an r-factorization and are still unique up to rearrangement and associates, which contradicts the fact that $R$ is an r-FFR.

(iii) Let $a \in \operatorname{Reg}(R)^{\#}$. Every $\tau$-r-divisor divisor is a regular divisor of $a$, so there can only be finitely many up to associate. 
(iv) Suppose we have an infinite sequence $\left\{a_{i}\right\}_{i=1}^{\infty}, a_{k} \in \operatorname{Reg}(R)^{\#}$ for all $k$ with $\left.a_{n+1}\right|_{\tau} a_{n}$ but $a_{n+1} \nsim a_{n}$ for all $n \geq 1$. But then we still have $\left.a_{n+1}\right|_{\tau} a_{n}, a_{k} \in \operatorname{Reg}(R)^{\#}$ for all $k$ but $a_{n+1} \not a_{n}$, so we contradict r-ACCP, concluding the proof.

Corollary 5.3. The r-UFRs, r-FFRs, r-HFRs and r-BFRs as defined in [1, Section 5] satisfy $\mathrm{r}-\mathrm{ACCP}$, and therefore $\tau$-r-ACCP. Hence, for $\tau$ refinable, each is $\tau$-r-atomic by Theorems 3.4 and 5.2 .

The following diagram summarizes our results ( $\nabla$ represents $\tau$ refinable):

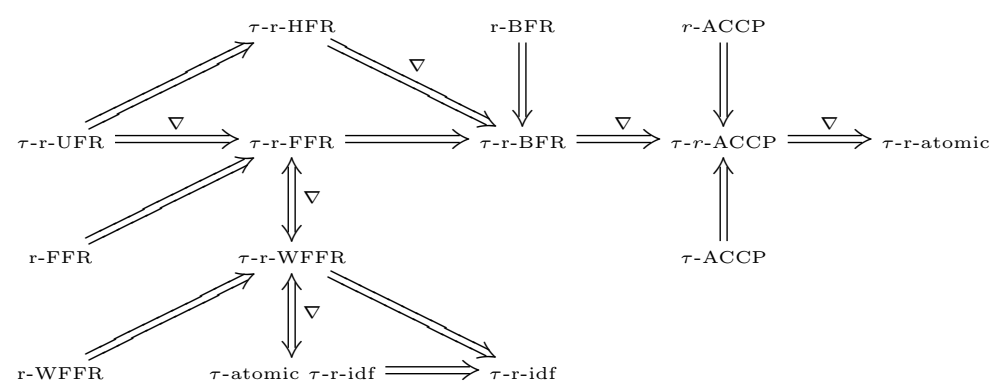

Lemma 5.4. Let $R$ be a commutative ring with 1 , and let $\tau$ be a symmetric relation on $R^{\#}$. Let $\alpha \in\{\emptyset$, atomic, strongly atomic, $m$-atomic, unrefinably atomic, very strongly atomic $\}$. Every non-unit element in $a \tau_{\text {reg- }} U$ - $\alpha$-factorization is an essential divisor. Moreover, given a $\tau_{\text {reg- }}$ $\alpha$-factorization, every $\tau$-factor is essential. When $\alpha=\emptyset$, we mean simply a $\tau_{\text {reg-U-factorization. }}$.

Proof. Let $a \in R$ be a non-unit, and let

$$
a=\lambda a_{1} \cdots a_{n}\left\lceil b_{1} \cdots b_{m}\right\rceil
$$

be a $\tau_{\text {reg }}-\mathrm{U}-\alpha$-factorization. Then

$$
a=\lambda a_{1} \cdots a_{n} b_{1} \cdots b_{m}
$$

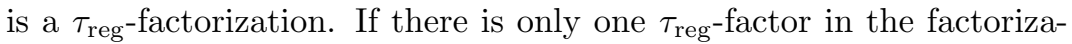
tion, i.e., $m+n=1$, then this factor is certainly essential. If it were removed, then it would imply that $a$ were a unit, a contradiction. We 
now may assume that $m+n \geq 2$, and therefore

$$
a=\lambda a_{1} \cdots a_{n} b_{1} \cdots b_{m}
$$

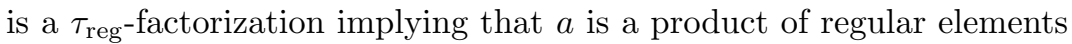
and hence is regular. Moreover, we have $(a)=\left(b_{1} \cdots b_{m}\right)$ so $a r=$ $b_{1} \cdots b_{m}$ for some $r \in R$. Hence,

$$
a=\lambda a_{1} \cdots a_{n} \cdot a \cdot r
$$

and $a$ is regular so cancellation implies that

$$
1=\lambda a_{1} \cdots a_{n} \cdot r,
$$

and in particular $a_{i} \in U(R)$ for all $1 \leq i \leq n$. Hence, there can be no non-unit inessential $\tau_{\text {reg-divisors as desired. }}$

Given a $\tau_{\text {reg- }}-\alpha$-factorization of a non-unit $a \in R$, say $a=\lambda a_{1} \cdots a_{n}$, we show that $a_{i}$ is essential for each $1 \leq i \leq n$. If $n=1$, this is immediate as above. Thus, $n \geq 2$, and therefore, $a_{i}$ is regular for each $1 \leq i \leq n$. Suppose for a moment that $a_{i}$ were not essential. Then

$$
(a)=\left(a_{1} \cdots a_{i-1} \widehat{a_{i}} a_{i+1} \cdots a_{n}\right)=\left(a_{1} \cdots a_{n}\right) .
$$

But this means that there is an $r \in R$ such that

$$
a_{1} \cdots a_{i-1} \widehat{a_{i}} a_{i+1} \cdots a_{n}=r \cdot a_{1} \cdots a_{n} .
$$

After canceling common factors, since each element on the left is regular, we see that $1=r \cdot a_{i}$, which means $a_{i} \in U(R)$, a contradiction since each $a_{j} \in R^{\#}$ for all $1 \leq j \leq n$. Thus, $a_{i}$ is essential for each $1 \leq i \leq n$ and $\lambda\left\lceil a_{1} \cdots a_{n}\right\rceil$ is indeed a $\tau$-U- $\alpha$ factorization.

The consequence of this lemma is that we see that $\tau_{\text {reg }}-\alpha$-factorizations and $\tau_{\text {reg }}-\mathrm{U}-\alpha$-factorizations coincide and we see there is a correspondence between the sets, given by the map

$$
\phi:\left\{\tau_{\text {reg- }}-\mathrm{U}-\alpha \text {-factorizations }\right\} \longrightarrow\left\{\tau_{\text {reg }}-\alpha \text {-factorizations }\right\}
$$

is given by

$$
\lambda a_{1} \cdots a_{n}\left\lceil b_{1} \cdots b_{m}\right\rceil \longmapsto\left(\lambda a_{1} \cdots a_{n}\right) b_{1} \cdots b_{m},
$$

and the inverse

$$
\phi^{-1}:\left\{\tau_{\text {reg- }}-\alpha \text {-factorizations }\right\} \longrightarrow\left\{\tau_{\text {reg }}-\mathrm{U}-\alpha \text {-factorizations }\right\}
$$


is given by

$$
\lambda a_{1} \cdots a_{n} \longmapsto \lambda\left\lceil a_{1} \cdots a_{n}\right\rceil
$$

This observation allows us to further consolidate many of our finite factorization properties when it comes to regular factorization. In particular, we formalize this by way of the following result.

Theorem 5.5. Let $R$ be a commutative ring with 1 , and let $\tau$ be a symmetric relation on $R^{\#}$. Let $\tau_{\text {reg }}:=\tau \cap(\operatorname{Reg}(R) \times \operatorname{Reg}(R))$. Let

$\alpha \in\{$ atomic, strongly atomic, m-atomic, unrefinably atomic, very strongly atomic $\}$

and

$\beta \in\{$ associate, strongly associate, very strongly associate $\}$.

Then, for any choice of $\alpha$ and $\beta$, we have the following:

(i) $R$ is $\tau_{\text {reg }}-\mathrm{U}-\alpha$ if and only if $R$ is $\tau_{\text {reg }}-\alpha$.

(ii) $R$ satisfies $\tau_{\text {reg-U-ACCP }}$ if and only if $R$ satisfies $\tau_{\text {reg }}$-ACCP.

(iii) $R$ is a $\tau_{\text {reg- }}-\mathrm{U}-\alpha-\beta-\mathrm{UFR}$ if and only if $R$ is a $\tau_{\text {reg }}-\alpha-\beta-\mathrm{UFR}$.

(iv) $R$ is a $\tau_{\text {reg-U- }-\alpha-H F R}$ if and only if $R$ is a $\tau_{\text {reg }}-\alpha-\mathrm{HFR}$.

(v) $R$ is a $\tau_{\text {reg- }}-\mathrm{U}-\mathrm{BFR}$ if and only if $R$ is a $\tau_{\text {reg-BFR. }}$.

(vi) $R$ is a $\tau_{\text {reg }}-\mathrm{U}-\alpha-\beta$-df ring if and only if $R$ is a $\tau_{\text {reg }}-\alpha-\beta$-df ring.

(vii) $R$ is a $\tau_{\text {reg }}-\mathrm{U}-\alpha, \tau_{\text {reg }}-\mathrm{U}-\alpha-\beta$-df ring if and only if $R$ is a $\tau_{\text {reg }}-\alpha$, $\tau_{\text {reg }}-\alpha-\beta$-df ring.

(viii) $R$ is a $\tau_{\text {reg- }}-\mathrm{U}-\beta-\mathrm{WFFR}$ if and only if $R$ is a $\tau_{\text {reg }}-\beta$-WFFR.

(ix) $R$ is a $\tau_{\text {reg }}-\mathrm{U}-\beta-\mathrm{FFR}$ if and only if $R$ is a $\tau_{\text {reg }}-\beta$-FFR.

If $\tau$ is refinable, then (vii) $\Leftrightarrow$ (viii) $\Leftrightarrow$ (ix).

Proof. (i) $(\Rightarrow)$. Let $a \in R$ be a non-unit. Then there is a $\tau_{\text {reg-U- }}$ $\alpha$ factorization of $a$, by Lemma 5.4. This factorization is of the form $a=\lambda\left\lceil a_{1} \cdots a_{n}\right\rceil$. By definition, $a=\lambda a_{1} \cdots a_{n}$ is a $\tau_{\text {reg-factorization }}$ and $a_{i}$ is $\tau_{\text {reg }}-\alpha$ for each $1 \leq i \leq n$, and therefore, this is a $\tau_{\text {reg }}-\alpha$ factorization of $a$.

$(\Leftarrow)$. This is shown in [43, Theorem 4.3].

(ii) $(\Rightarrow)$. Let $a \in R$ be a non-unit. Suppose there was an ascending chain of principal ideals of the form $(a) \subsetneq\left(a_{1}\right) \subsetneq\left(a_{2}\right) \subsetneq \cdots$, such that 


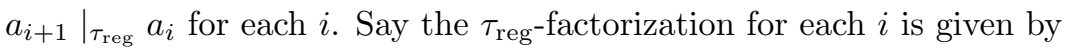

$$
a_{i}=\lambda a_{i+1} a_{i 1} \cdots a_{i n_{i}}
$$

because $\left(a_{i}\right) \subsetneq\left(a_{i+1}\right)$, we know that this $\tau_{\text {reg-factorization is non- }}$

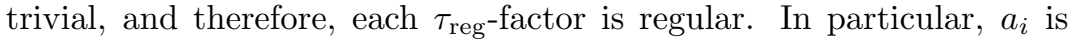
regular, and therefore by Lemma 5.4, is essential. This would contradict the fact that $R$ satisfies $\tau_{\text {reg }}-\mathrm{U}-\mathrm{ACCP}$.

$(\Leftarrow)$. This is shown in [43, Theorem 4.3].

(iii) (respectively (iv)). Let $a \in R$ be a non-unit. Then, by Lemma 5.4, $a$ has a $\tau_{\text {reg-U- }} \alpha$ factorization if and only if $a$ has a $\tau_{\text {reg- }}{ }^{-}$ $\alpha$-factorization. Furthermore, since the $\tau_{\text {reg- }}$ U-factorizations have no inessential divisors, it is clear that the equivalence of the uniqueness (respectively constant length) of these factorizations follows as well.

(v) and (ix). Let $a \in R$ be a non-unit. By Lemma 5.4, the correspondence shows that we may apply $\phi^{-1}$ to any $\tau_{\text {reg-factorization }}$ of $a$ of length $n$ and get a $\tau_{\text {reg- }}$ - factorization with the same $n \tau_{\text {reg }^{-}}$ factors all occurring as the $\tau_{\text {reg }}$ essential divisors in the corresponding

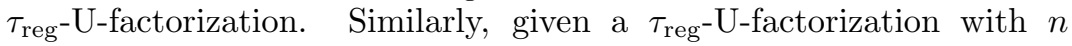
essential divisors, we may apply $\phi$ to this factorization and get a $\tau_{\text {reg }^{-}}$ factorization of length $n$ with the same $\tau_{\text {reg-factors as the essential }}$

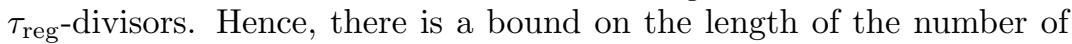
essential divisors in any $\tau_{\text {reg- }} \mathrm{U}$-factorization of $a$ if and only if there is

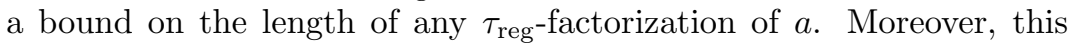
same correspondence shows that there are the same number of $\tau_{\mathrm{reg}^{-}}$ factorizations of $a$ up to $\beta$ as there are $\tau_{\text {reg-U-factorizations of } a \text { up }}$ to $\beta$.

(vi) (respectively (viii)). Let $a \in R$ be a non-unit. As in the proof of (v) and (ix), it is clear that the set of $\tau_{\text {reg-divisors and essential }} \tau_{\text {reg- }}{ }^{-}$ divisors of $a$ are the same by the correspondence given in Lemma 5.4

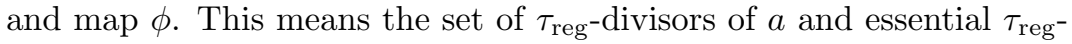
divisors of $a$ up to $\beta$ are the same. Moreover, this also means that the set of $\tau_{\text {reg }}-\alpha$ divisors and the set of $\tau_{\text {reg }}-\alpha$-essential divisors are the same up to $\beta$ as well.

(vii). This follows immediately by combining the results of parts (i) and (vi). 
We can further relate the various properties by removing the very strongly atomic choice for $\alpha$ and the very strongly associate choice for $\beta$ in Theorem 5.5. The results are combined below.

Corollary 5.6. Let $R$ be a commutative ring with 1 , and let $\tau$ be a symmetric relation on $R^{\#}$. Let $\tau_{\text {reg }}:=\tau \cap(\operatorname{Reg}(R) \times \operatorname{Reg}(R))$. Let

$\alpha \in\{$ atomic, strongly atomic, m-atomic, unrefinably atomic $\}$

and

$\beta \in\{$ associate, strongly associate $\}$.

Then, for any choice of $\alpha$ and $\beta$, we have the following:

(i) $R$ is $\tau_{\text {reg }}-\mathrm{U}-\alpha$ if and only if $R$ is $\tau_{\text {reg }}-\alpha$ if and only if $R$ is $\tau$ regular-atomic.

(ii) $R$ satisfies $\tau_{\text {reg-U-ACCP }}$ if and only if $R$ satisfies $\tau_{\text {reg }}$-ACCP if and only if $R$ satisfies $\tau$-regular-ACCP.

(iii) $R$ is a $\tau_{\text {reg }}-\mathrm{U}-\alpha-\beta$-UFR if and only if $R$ is a $\tau_{\text {reg }}-\alpha-\beta$-UFR if and only if $R$ is $\tau$-regular-UFR.

(iv) $R$ is a $\tau_{\text {reg }}-\mathrm{U}-\alpha$-HFR if and only if $R$ is a $\tau_{\text {reg }}-\alpha$-HFR if and only if $R$ is $\tau$-regular-HFR.

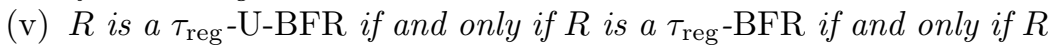
is $\tau$-regular-BFR.

(vi) $R$ is a $\tau_{\text {reg }}-\mathrm{U}-\alpha-\beta$-df ring if and only if $R$ is a $\tau_{\text {reg }}-\alpha-\beta$-df ring if and only if $R$ is a $\tau$-regular-idf ring.

(vii) $R$ is a $\tau_{\text {reg }}-\mathrm{U}-\alpha, \tau_{\text {reg }}-\mathrm{U}-\alpha-\beta$-df ring if and only if $R$ is a $\tau_{\text {reg }}-\alpha$, $\tau_{\text {reg }}-\alpha-\beta$-df ring if and only if $R$ is $\tau$-regular-atomic, $\tau$-regular-idf ring.

(viii) $R$ is a $\tau_{\text {reg }}-\mathrm{U}-\beta$-WFFR if and only if $R$ is a $\tau_{\text {reg }}-\beta$-WFFR if and only if $R$ is $\tau$-regular-WFFR.

(ix) $R$ is a $\tau_{\text {reg }}-\mathrm{U}-\beta$-FFR if and only if $R$ is a $\tau_{\text {reg }}-\beta$-FFR if and only if $R$ is $\tau$-regular-FFR.

If $\tau$ is refinable, then (vii) $\Leftrightarrow$ (viii) $\Leftrightarrow$ (ix).

Proof. The first equivalence in each statement (i) for $1 \leq i \leq 9$ follows directly from Theorem 5.5. Similarly, the second equivalence in each statement (i) for $1 \leq i \leq 9$ follows from Theorem 4.8. 
We conclude the article with a diagram which summarizes many of the equivalences and relationships demonstrated thus far where $\tau_{\text {reg }}$ is defined as above,

$\alpha \in\{$ atomic, strongly atomic, m-atomic, unrefinably atomic $\}$,

$\beta \in\{$ associate, strongly associate $\}$,

and $\nabla$ represents $\tau$ refinable.

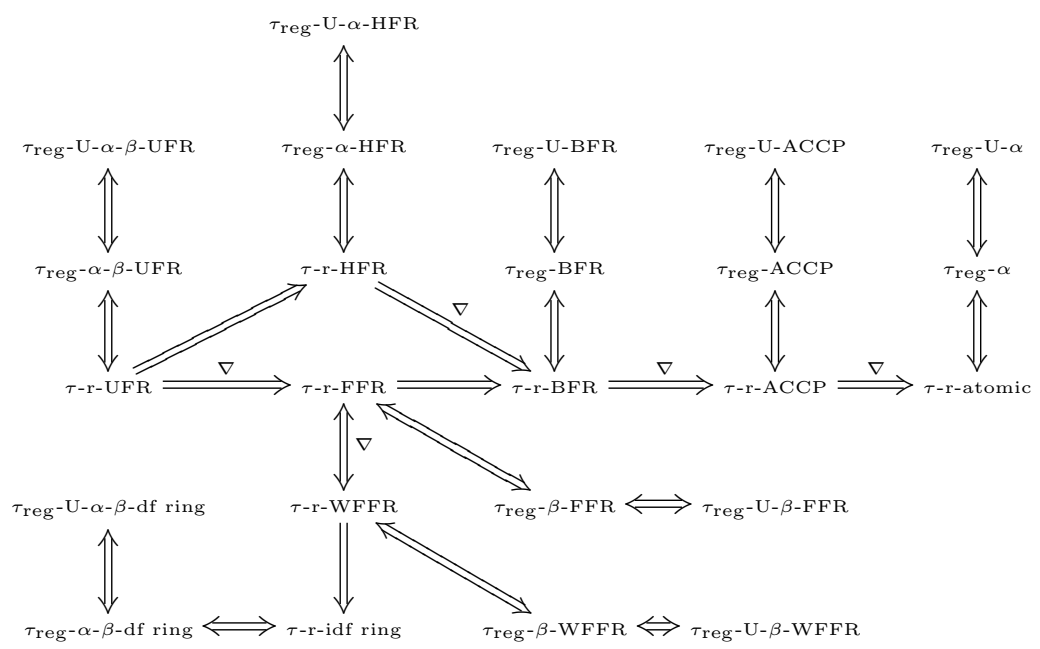

6. Analysis of two explicit rings and further questions. In this section, we explore some rings which have been studied extensively in the literature to see which of the $\tau$-r-finite factorization properties these rings satisfy. Many authors have pursued the theory of factorization in integral domains by way of ideal theory. In the case of integral domains studying factorization from an element perspective is the same as studying the principal ideal structure. When dealing with commutative rings with zero-divisors, these two approaches diverge. There is a substantial amount of research done on ideal theoretic properties of commutative rings with zero-divisors. For instance, the monograph of Huckaba [32] uses this approach. The theory of Krull, Mori, Prüfer, Marot, v-Marot, and C-rings has been studied extensively in $[18,19,20,30,31,34,35,36,37,38,39,40,45]$. 
We supply some results regarding the $\tau$-regular factorization properties of the two very explicit rings recently studied in $[\mathbf{2 1}, \mathbf{2 6}]$. We hope that this will serve as an example of the kinds of questions we hope to eventually be able to answer about a larger class of rings that have been of interest over the years by many authors studying commutative rings with zero-divisors.

We begin with the self-idealization of a principal ideal domain (PID) studied in [21]. Let $D$ be a PID, and let

$$
R(D)=\left\{\left(\begin{array}{ll}
a & b \\
0 & a
\end{array}\right) \mid a, b \in D\right\} .
$$

Then, it is well known that $R(D)$ is a Noetherian commutative ring with identity. We include definitions in the theorem for convenience for the reader. An irreducible or atom is a non-zero non-unit such that $a=b c$ implies $(a)=(b)$ or $(a)=(c)$. Moreover, $a, b \in R$ are said to be associates if $(a)=(b) . \quad R$ is said to be atomic if every non-zero non-unit has a factorization into irreducible elements. Chang and Smertnig further investigate factorization properties of these rings, which we summarize below.

Theorem 6.1. ([21, Corollary 9]). Let $D$ be a PID. Then we have the following:

(i) $R(D)$ is Noetherian.

(ii) $R(D)$ is a $\mathrm{BFR}$ ( $R$ is atomic and there is a bound on the length of irreducible factorizations of every non-zero non-unit of $R$ ).

(iii) $R(D)$ is an FFR ( $R$ is atomic and each nonzero non-unit has only nitely many factorizations into irreducibles, up to order and associates) if and only if $D / p D$ is finite for all prime elements $p \in D$.

(iv) If $D$ is a field, then every non-zero non-unit of $R(D)$ is a prime, and hence $R(D)$ is a UFR ( $R$ is atomic and every non-zero principal ideal of $R$ can be written as a product of principal prime ideals) with a unique nonzero (prime) ideal.

We will use these observations to give examples of rings which will satisfy various $\tau$-regular finite factorization properties studied in 
the present article as well as other $\tau$-finite factorization properties previously studied in the literature.

Corollary 6.2. Let $D$ be a PID, and let $\tau$ be any symmetric relation on $R(D)^{\#}$. Let $\tau_{\text {reg }}:=\tau \cap(\operatorname{Reg}(R) \times \operatorname{Reg}(R))$. Let

$\alpha \in\{$ atomic, strongly atomic, m-atomic, unrefinably atomic $\}$

and

$\beta \in\{$ associate, strongly associate $\}$.

Then, for any choice of $\alpha$ and $\beta$, we have the following:

(i) $R(D)$ satisfies $\tau$-ACCP, $\tau$-U-ACCP and $\tau$-r-ACCP (equivalently,

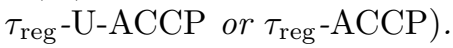

(ii) If $\tau$ is refinable, then $R(D)$ is $\tau$-atomic, $\tau$-U-atomic, and $\tau$-ratomic (equivalently, $\tau_{\text {reg }}-\mathrm{U}-\alpha$ or $\tau_{\text {reg }}-\alpha$ ).

(iii) $R(D)$ is a $\tau$-BFR, $\tau$-U-BFR, $\tau$-r-BFR (equivalently, $\tau_{\text {reg-U-BFR }}$ or $\left.\tau_{\text {reg }}-\mathrm{BFR}\right)$.

(iv) If $D / p D$ is finite for all prime elements $p \in D$, then $R(D)$ is a $\tau$-associate-FFR, $\tau$-U-associate-FFR and $\tau$-r-FFR (equivalently, $\tau_{\text {reg }}-\mathrm{U}-\beta-\mathrm{FFR}$ or $\tau_{\text {reg }}-\beta$-FFR).

(v) If $D$ is a field, the $R(D)$ is a $\tau$-atomic-associate-UFR, $\tau$-Uatomic-associate-UFR, $\tau$-r-UFR (equivalently, $\tau_{\text {reg }}-\mathrm{U}-\alpha-\beta$-UFR or $\left.\tau_{\text {reg }}-\alpha-\beta-\mathrm{UFR}\right)$.

Proof. We begin by noting the equivalent part of each of the above follows directly from Corollary 5.6.

(i) Since $R(D)$ is Noetherian by Theorem $6.1, R(D)$ satisfies ACCP. It was shown in [42, Theorem 4.1] that ACCP implies $\tau$-ACCP for any $\tau$. By [43, Theorem 4.3], $\tau$-ACCP implies $\tau$-U-ACCP. Finally, by Theorem 5.1, we see that $\tau$-ACCP implies $\tau$-r-ACCP.

(ii) By [42, Theorem 4.1], since $\tau$ is assumed to be refinable, $\tau$ ACCP implies $\tau$-atomic. By [43, Theorem 4.3], we have $\tau$-atomic implies $\tau$-U-atomic. Finally, by Theorem 5.1, $\tau$-atomic implies $\tau$-ratomic.

(iii) $R(D)$ is a BFR which satisfies ACCP. $R(D)$ satisfies ACCP, so certainly $R(D)$ is atomic. An atomic ring which has a bound on the length of atomic factorizations of every non-zero non-unit certainly has 
a bound on the length of all factorizations. We can simply use the same bound since given any factorization, we could replace the factors with atomic factorizations and get a possibly even longer factorization which is atomic. Hence, we can be sure $R(D)$ satisfies the notion of BFR used in [42], where there is a bound on the length of all factorizations of each non-zero non-unit. Let $a \in R(D)$ be a non-zero, non-unit. Any $\tau$-factorization of $a$ is certainly a factorization of $a$, so we may again use the same bound and this proves $R(D)$ is a $\tau$-BFR. By [43, Theorem 4.3], $\tau$-BFR implies $\tau$-U-BFR and, by Theorem 5.1, we see that $\tau$-BFR implies $\tau$-r-BFR.

(iv) We again point out that an atomic FFR using the definition from [21] coincides with the definition used in [42] since any factorization can be refined into an atomic factorization. Thus, given any non-zero non-unit $a \in R$, the set of atomic factorizations of $a$ up to rearrangement and associate is finite if and only if the set of factorizations of $a$ is finite. If $D / p D$ is finite for all prime elements $p \in D$, then $R(D)$ is an FFR. For every non-zero non-unit $a \in R(D)$, there are a finite number of factorizations of $a$ up to rearrangement and associate. Every $\tau$-factorization of $a$ is certainly among the factorizations of $a$, and hence the set of $\tau$-factorizations up to rearrangement and associate is finite proving $R(D)$ is a $\tau$-associate-FFR. By [43, Theorem 4.3], we have $\tau$-associate-FFR implies $\tau$-U-associate-FFR. By Theorem 5.1, we see that $\tau$-associate-FFR implies $\tau$-r-FFR.

(v) If $D$ is a field, then $R(D)$ is a UFR. In general, this is not necessarily enough to conclude that $R$ is a $\tau$-atomic-associate-UFR; however, in this case, we actually have a stronger condition that every non-zero, non-unit is prime. Prime implies irreducible, but not necessarily any of the stronger forms of irreducible. We show that irreducible implies $\tau$-irreducible. Let $a=\lambda a_{1} \cdots a_{n}$ be a $\tau$ factorization. Then this is a factorization, so $a \sim a_{i}$ for some $1 \leq i \leq n$. Hence $a$ is $\tau$-atomic. This shows that every non-zero non-unit of $a \in R(D)$ is a $\tau$-irreducible element. This means $a=1 \cdot a$ is a $\tau$ atomic factorization of $a$. This is also an atomic factorization. $R(D)$ is known to be a UFR. Suppose there were another $\tau$-irreducible factorization of $a$, say $a=\lambda b_{1} \cdots b_{m}$. Every $b_{i}$ is a non-zero, nonunit of $R(D)$, and therefore prime and irreducible. This would yield a distinct irreducible factorization of $a$, contradicting the fact that $R(D)$ is an atomic-associate-UFR, a contradiction. This proves $R(D)$ is a $\tau$ - 
atomic-associate-UFR. Since every $\tau$-factorization has length 1 , we can also be sure that every $\tau$-factor is essential, and therefore a $\tau$-atomicU-factorization. This proves $R(D)$ is a $\tau$-atomic-associate-UFR. By Theorem 5.1, we see that $\tau$-atomic-associate-UFR implies $\tau$-r-UFR, completing the proof.

Corollary 6.3. Let $D$ be a field, and let $\tau$ be a symmetric relation on $R(D)^{\#}$. Let

$\alpha \in\{$ atomic, strongly atomic, m-atomic, unrefinably atomic $\}$

and

$\beta \in\{$ associate, strongly associate $\}$.

Then, for any choice of $\alpha$ and $\beta$, we have the following:

(i) $R(D)$ is a $\tau$-r-UFR (respectively $\tau_{\text {reg }}-\mathrm{U}-\alpha-\beta$-UFR, $\tau_{\text {reg }}-\alpha-\beta$-UFR, $\tau$-atomic-associate-UFR, $\tau$-U-atomic-associate-UF).

(ii) $R(D)$ is a $\tau$-r-HFR (respectively $\tau_{\text {reg }}-U$ - $\alpha$-HFR, $\tau_{\text {reg }}-\alpha-\mathrm{HFR}, \tau$ atomic-HFR, $\tau$-U-atomic-HFR).

(iii) $R(D)$ is a $\tau$-r-BFR (respectively $\tau_{\text {reg }}-\mathrm{U}-\mathrm{BFR}, \tau_{\text {reg }}$-BFR, $\tau$-BFR, $\tau$-U-BFR).

(iv) $R(D)$ is a $\tau$-r-FFR (respectively $\tau_{\text {reg }}-\mathrm{U}-\beta$-FFR, $\tau_{\text {reg }}-\beta$-FFR, $\tau$ associate-FFR, $\tau$-U-associate-FFR).

(v) $R(D)$ is a $\tau$-r-WFFR (respectively $\tau_{\text {reg }}-\mathrm{U}-\beta$-WFFR, $\tau_{\text {reg }}-\beta$-WFFR, $\tau$-associate-WFFR, $\tau$-U-associate-WFFR).

(vi) $R(D)$ is a $\tau$-r-atomic (respectively $\tau_{\text {reg }}-\mathrm{U}-\alpha, \tau_{\text {reg }}-\alpha$, $\tau$-atomic, $\tau$ $\mathrm{U}$-atomic) $\tau$-r-idf ring (respectively $\tau_{\text {reg }}-\mathrm{U}-\alpha-\beta$-df ring, $\tau_{\text {reg }}-\alpha-$ $\beta$-df ring, $\tau$-atomic-associate-idf ring, $\tau$-U-atomic-associate-idf ring).

(vii) $R(D)$ satisfies $\tau$-r-ACCP (respectively $\tau_{\text {reg-U-ACCP, }} \tau_{\text {reg }}$-ACCP, $\tau$-ACCP, $\tau$-U-ACCP).

(viii) $R(D)$ is $\tau$-r-atomic (respectively $\tau_{\text {reg }}-\mathrm{U}-\alpha, \tau_{\text {reg }}-\alpha$, $\tau$-atomic, $\tau$-Uatomic).

Proof. These are immediate from the observation in Corollary 6.2 (v) as well as Corollary 5.6.

We now turn our attention to the $\operatorname{ring} \mathbb{Z}_{p^{n}}[X]$ which was studied extensively in [26]. In this article the authors choose the definitions 
weakly irreducible and weakly associate for what we have called irreducible and associate, respectively. They use the terms irreducible and associate for what we have called strongly irreducible and strongly associate, respectively. We will soon see that the ring in question is présimplifiable and therefore certainly strongly associate, and our definitions will be equivalent (in fact, very strongly atomic, unrefinably atomic and m-atomic are also equivalent here), so there will be no confusion for $\mathbb{Z}_{p^{n}}[X]$.

Many of the key factorization properties of this ring derive from the following fact [26, Fact 1.5], where $\mathrm{Nil}(R)$ is the nilradical, $J(R)$ is the Jacobson radical and $Z(R)$ is the set of zero-divisors.

$$
\operatorname{Nil}\left(\mathbb{Z}_{p^{n}}[X]\right)=J\left(\mathbb{Z}_{p^{n}}[X]\right)=(p)=Z\left(\mathbb{Z}_{p^{n}}[X]\right) .
$$

If the zero-divisors are contained in the Jacobson radical, then the ring is présimplifiable. This is discussed in many sources, but for $\tau$-factorization, the author suggests [42, Lemma 2.2] and [44]. As mentioned following Theorem 2.2, the présimplifiable property has the effect of making all of the associate relations coincide, which in turn makes irreducible and very strongly irreducible (hence everything in between) as well as $\tau$-irreducible and $\tau$-very strongly irreducible equivalent. As in [43, Theorem 3.5], this also has the added benefit of making every non-unit $\tau$-factor essential, so $\tau$-U-factorizations will also coincide with the usual factorizations.

We now summarize several of the results about $\mathbb{Z}_{p^{n}}[X]$ from $[\mathbf{2 6}]$ which will be of interest in order to draw conclusions about which $\tau$ regular finite factorization properties this ring will satisfy.

Theorem 6.4 ([26]). Let $R=\mathbb{Z}_{p^{n}}[X]$. Then we have the following:

(i) $R$ is Noetherian,

(ii) $R$ is atomic,

(iii) $R$ has infinite elasticity,

(iv) each regular element of $R$ has a finite number of factorizations into irreducibles.

These results will allow us to answer several questions about the factorization properties discussed in this article. 
Theorem 6.5. Let $R=\mathbb{Z}_{p^{n}}[X]$, and let $\tau$ be a symmetric relation on $R^{\#}$. Let

$\alpha \in\{$ atomic, strongly atomic, m-atomic, unrefinably atomic, very strongly atomic $\}$,

and let

$\beta \in\{$ associate, strongly associate, very strongly associate $\}$.

(i) $R$ satisfies $\tau$-ACCP, $\tau$-U-ACCP, $\tau$-r-ACCP and $\tau_{\text {reg }}-\mathrm{ACCP}$.

(ii) $R$ is $\mathrm{r}$-atomic, and if $\tau$ is refinable, then $R$ is $\tau$ - $\alpha, \tau$ - $\mathrm{U}-\alpha, \tau$-ratomic, $\tau_{\text {reg }}-\alpha$ and $\tau_{\text {reg }}-\mathrm{U}-\alpha$.

(iii) $R$ is an r-FFR, $\tau$-r-FFR, $\tau_{\text {reg }}-\beta$-FFR and $\tau_{\text {reg }}-\mathrm{U}-\beta$-FFR.

(iv) $R$ is an $\mathrm{r}$-WFFR, $\tau$-r-WFFR, $\tau_{\text {reg }}-\beta$-WFFR and $\tau_{\text {reg }}-\mathrm{U}-\beta$-WFFR.

(v) $R$ is an r-idf ring, $\tau$-r-idf ring, $\tau_{\text {reg }}-\mathrm{U}-\alpha-\beta$-df ring and $a \tau_{\text {reg }}-\alpha-$ $\beta$-df ring.

(vi) $R$ is a $\mathrm{r}$-atomic $\mathrm{r}$-idf ring and, if $\tau$ is refinable, then $R$ is a $\tau$-ratomic $\tau$-r-idf ring, a $\tau_{\text {reg }}-\mathrm{U}-\alpha \tau_{\text {reg }}-\mathrm{U}-\alpha-\beta$-df ring, and a $\tau_{\text {reg }}-\alpha$ $\tau_{\text {reg }}-\alpha-\beta$-df ring.

Proof. We begin by noting that, since $R$ is présimplifiable, all the associate relations and irreducibles coincide. This allows us to add very strongly associate and very strongly atomic to the choices of $\alpha$ and $\beta$

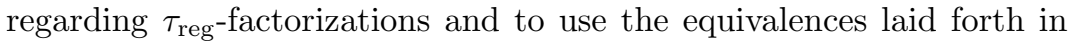
Corollary 5.6.

The proof of (i) and (ii) follows from the same argument as for (i) and (ii) from Corollary 6.2 and from the fact that $R$ is Noetherian.

(iii) Let $a \in \operatorname{Reg}(R)^{\#}$. Then, since we know $R$ is atomic and there are finitely many irreducible factorizations of $a$ up to rearrangement and associate from Corollary 6.4, we can be sure that there are only finitely many factorizations of $a$ up to rearrangement and associate. This makes $R$ an r-FFR. If there are finitely many regular factorizations of $a$, then we can be sure that $R$ is also a $\tau$-r-FFR since every $\tau$-r-factorization is among the finitely many r-factorizations (up to rearrangement and associate).

(iv) Since we have established that $R$ is an r-FFR, there are only finitely many factorizations of any $a \in \operatorname{Reg}(R)^{\#}$ up to rearrangement 
and associates. This means there are only finitely many divisors of $a$ up to associate, making $R$ an r-WFFR. If there are only finitely many divisors of $a$ up to associate, then there are certainly only finitely many $\tau$-divisors of $a$ up to associate, making $R$ a $\tau$-r-WFFR.

(v) Let $a \in \operatorname{Reg}(R)^{\#}$. Then $a$ has a finite number of factors and $\tau$-factors up to associate. Then $a$ certainly has a finite number of irreducible divisors and $\tau$-irreducible $\tau$-divisors up to associate. Thus, $R$ is both an r-idf ring and a $\tau$-r-idf ring.

(vi) This follows immediately from (ii) and (v).

As a consequence of Theorem 6.4 (iii), we have the following.

Corollary 6.6. Let $R=\mathbb{Z}_{p^{n}}[X]$, and let $\tau$ be a symmetric relation on $R^{\#}$. Then:

(i) $R$ is neither an HFR nor a UFR.

(ii) $R$ need not be a $\tau$-HFR, $\tau$-U-HFR, $\tau$-UFR nor a $\tau$-U-UFR.

Proof.

(i) By Theorem 6.4 (iii), we know that $R$ has infinite elasticity. As in [26], this means that there are non-zero, non-unit elements $a \in R$ such that $a$ can be factored into a product of irreducible elements of different lengths. This forces $R$ to fail to be an HFR or a UFR.

(ii) Because $R$ is présimplifiable, $\tau$-factorizations and $\tau$-U-factorizations coincide since every factor is essential. If we were to set $\tau_{d}:=R^{\#} \times R^{\#}$, then we would get the usual factorizations. This would yield an example of a $\tau$ for which $R$ is neither $\tau$-HFR, $\tau$-U-HFR, $\tau$-UFR nor $\tau$-U-UFR.

Our hope is that this type of analysis could be performed on the enormous class of rings that have been studied by many authors mentioned in the introduction to this section. It would be fascinating to know which of the various classes of rings satisfy the equivalences given in the present paper in a similar fashion as above. As one can see by only looking at these two very explicit rings in this section, there were a number of results regarding their regular factorization and $\tau$-regular factorization properties. 
Many of the major breakthroughs in factorization in integral domains have come through studying ideal theory. In the integral domain case, studying factorization through principal ideals and elements coincide; however, this is not the case in rings with zerodivisors. Many authors have sought to study factorization in rings with zero-divisors by way of generalizing various types of rings defined through their ideal theory. To name a few, we have Krull, Mori, Prüfer, Marot, v-Marot, and C-rings, which been studied extensively in $[18,19,20,30,31,34,35,36,37,38,39,40,45]$.

Many of these rings are well studied in the case of integral domains; however, as is often the case when extending definitions to rings with zero-divisors, many formerly equivalent definitions diverge. When dealing with regular elements as in the present article, there is hope that many of these definitions may again return to being equivalent, as we have seen thus far. It would be interesting for future research to answer many of the questions which arise about which of the various equivalences laid forth in this article will hold for the different types of rings mentioned above. Moreover, as suggested by the referee, much of this work could be reframed in the context of cancellative semigroups where research abounds in the literature. This may help to connect the present research with much of the research done on Krull rings. Unfortunately, the author fears the scope of the aforementioned project is too large to take on in the present article.

Acknowledgments. The author would like to acknowledge both The University of Iowa and Viterbo University for their support while the research involved was completed during employment at both universities. I would also like to thank the referee for catching many potentially embarrassing typographical errors and improving the proofs of several theorems throughout, especially for greatly improving the bibliography to include many excellent references related to this article and suggesting several ideas for future research in which the final section barely scratches the surface. The article has no doubt been substantially improved thanks to the diligent and careful work of the referee.

\section{REFERENCES}

1. Ahmet G. Ağargün, D.D. Anderson and Silvia Valdez-Leon, Factorization in commutative rings with zero divisors, III, Rocky Mountain J. Math. 31 (2001), $1-21$. 
2. M. Alan and A.G. Ağargün, Some factorization properties in commutative rings, Int. J. Pure Appl. Math. 18 (2005), 349-358.

3. D.D. Anderson, ed., Factorization in integral domains, Lect. Notes Pure Appl. Math. 189, Marcel Dekker, New York, 1997.

4. D.D. Anderson, D.F. Anderson and M. Zafrullah, Factorization in integral domains, J. Pure Appl. Alg. 69 (1990), 1-19.

5. D.D. Anderson, M. Axtell, S.J. Forman and Joe Stickles, When are associates unit multiples?, Rocky Mountain J. Math. 34 (2004), 811-828.

6. D.D. Anderson and S. Chun, Irreducible elements in commutative rings with zero-divisors, Rocky Mountain J. Math. 37 (2011), 741-744.

7. D.D. Anderson and Andrea M. Frazier, On a general theory of factorization in integral domains, Rocky Mountain J. Math. 41 (2011), 663-705.

8. D.D. Anderson and R. Markanda, Unique factorization in rings with zero divisors, Houston J. Math. 11 (1985), 15-30.

9. D.D. Anderson and Silvia Valdes-Leon, Factorization in commutative rings with zero divisors, Rocky Mountain J. Math. 26 (1996), 439-480.

10. - Factorization in commutative rings with zero divisors, II, in Factorization in integral domains, Lect. Notes Pure Appl. Math. 189, Dekker, New York, 1997.

11. M. Axtell, $U$-factorizations in commutative rings with zero divisors, Comm. Alg. 30 (2002), 1241-1255.

12. M. Axtell, S. Forman, N. Roersma and J. Stickles, Properties of $U$ factorizations, Inter. J. Comm. Rings 2 (2003), 83-99.

13. N.R. Baeth and R. Wiegand, Factorization theory and decomposition of modules, Amer. Math. Month. 120 (2013), 3-34.

14. A. Bouvier, Sur les anneaux de fractions des anneaux atomiques présimplifables, Bull. Sci. Math. 95 (1971), 371-376.

15. Anneaux présimplifables, C.R. Acad. Sci. Paris 274 (1972), A1605A1607.

16. _ Résultats nouveaux sur les anneaux présimplifables, C.R. Acad. Sci. Paris 275 (1972), A955-A957.

17. , Anneaux présimplifables, Rev. Roum. Math. Pures Appl. 19 (1974), $713-724$.

18. Gyu Whan Chang, Characterizations of a Krull ring $R[X]$, Bull. Korean Math. Soc. 28 (2001), 543-549.

19. Eakin-Nagata theorem for commutative rings whose regular ideals are finitely generated, Korean J. Math. 18 (2010), 271-275.

20. Gyu Whan Chang and Byung Gyun Kang, Integral closure of a ring whose regular ideals are finitely generated, J. Algebra 251 (2002), 529-537.

21. Gyu Whan Chang and D. Smertnig, Factorization in the self-idealization of a PID, Boll. Union. Mat. Ital. 9 (2013), 363-377. 
22. S.T. Chapman, ed., Arithmetical properties of commutative rings and monoids, Lect. Notes Pure Appl. Math. 241, Chapman \& Hall/CRC, Boca Raton, 2005.

23. L.E. Dickson, Finiteness of the odd perfect and primitive abundant numbers with distinct factors, Amer. J. Math. 35 (1913), 413-442.

24. C.R. Fletcher, Unique factorization rings, Math. Proc. Cambr. Philos. Soc. 65 (1969), 579-583.

25. The structure of unique factorization rings, Math. Proc. Cambr. Philos. Soc. 673 (1970), 535-540.

26. C. Frei and S. Frisch, Non-unique factorization of polynomials over residue class rings of the integers, Comm. Alg. 39 (2011), 1482-1490.

27. A. Geroldinger, Additive group theory and non-unique factorizations, in Combinatorial number theory and additive group theory, A. Geroldinger and I. Ruzsa, eds., Birkhäuser, Berlin, 2009.

28. A. Geroldinger and F. Halter-Koch, Non-unique factorizations. algebraic, combinatorial and analytic theory, Pure Appl. Math. 278, Chapman \& Hall/CRC, Boca Raton, 2006.

29. A. Geroldinger, S. Ramacher, and A. Reinhart, On v-marot mori rings and c-rings, http://arxiv.org/abs/1401.2761.

30. S. Glaz, Controlling the zero divisors of a commutative ring, in Commutative ring theory and applications, Lect. Notes Pure Appl. Math. 231, Marcel Dekker, New York, 2003.

31. F. Halter-Koch, Weak module systems and applications: a multiplicative theory of integral elements and the Marot property, in Commutative Ring Theory and Applications, Lect. Notes Pure Appl. Math. 231, Marcel Dekker, New York, 2003.

32. J.A. Huckaba, Commutative rings with zero-divisors, Pure Appl. Math. 117, Marcel Dekker, New York, 1988.

33. J.R. Juett, Generalized comaximal factorization of ideals, J. Algebra 352 (2012), 141-166.

34. Byung Gyun Kang, A characterization of Krull rings with zero divisors, J. Pure Appl. Alg. 72 (1991), 33-38.

35. , Integral closure of rings with zero-divisors, J. Alg. 162 (1993), 317323.

36. Characterizations of Krull rings with zero divisors, J. Pure Appl. Alg. 146 (2003), 283-290.

37. R.E. Kennedy, Krull rings, Pacific J. Math. 89 (1980), 131-136.

38. T.G. Lucas, The Mori property in rings with zero divisors, in Rings, modules, algebras, and Abelian groups, Lect. Notes Pure Appl. Math. 236, Marcel Dekker, New York, 2004.

39. Thomas G. Lucas, Krull rings, Prüfer v-multiplication rings and the ring of finite fractions, Rocky Mountain J. Math. 35 (2005), 1251-1325. 
40. Thomas G. Lucas, The Mori property in rings with zero divisors II, Rocky Mountain J. Math. 37 (2007), 1195-1228.

41. S. McAdam and R. Swan, Unique comaximal factorization, J. Alg. 276 (2004), 180-192.

42. C.P. Mooney, Generalized factorization in commutative rings with zerodivisors, Houston J. Math. 41 (2015), 15-32.

43. Christopher Park Mooney, Generalized u-factorization in commutative rings with zero-divisors, Rocky Mountain J. Math. 45 (2015), 637-660.

44. $\quad \tau$-complete factorization in commutative rings with zero-divisors, Houston J. Math. 42 (2016), 23-44.

45. E. Osmanagić, On an approximation theorem for Krull rings with zerodivisors, Comm. Alg. 27 (1999), 3647-3657.

Department of Mathematics and Physics, Westminster College, Fulton, MO 65251

Email address: christopher.mooney@westminster-mo.edu 